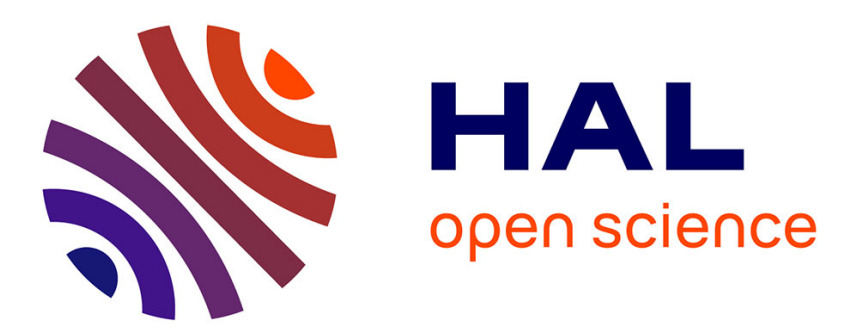

\title{
A parametric deformable model to fit unstructured 3D data
}

\author{
Eric Bardinet, Laurent D. Cohen, Nicholas Ayache
}

\section{To cite this version:}

Eric Bardinet, Laurent D. Cohen, Nicholas Ayache. A parametric deformable model to fit unstructured 3D data. Computer Vision and Image Understanding, 1998, 71 (1), pp.39-54. 10.1006/cviu.1997.0595 . inria-00615039

\section{HAL Id: inria-00615039 \\ https://hal.inria.fr/inria-00615039}

Submitted on 17 Aug 2011

HAL is a multi-disciplinary open access archive for the deposit and dissemination of scientific research documents, whether they are published or not. The documents may come from teaching and research institutions in France or abroad, or from public or private research centers.
L'archive ouverte pluridisciplinaire HAL, est destinée au dépôt et à la diffusion de documents scientifiques de niveau recherche, publiés ou non, émanant des établissements d'enseignement et de recherche français ou étrangers, des laboratoires publics ou privés. 


\title{
A Parametric Deformable Model To Fit Unstructured 3D Data
}

\author{
Eric BARDINET ${ }^{1}$,Laurent D. COHEN ${ }^{2}$, Nicholas AYACHE $^{1}$ \\ ${ }^{1}$ EPIDAURE Project, INRIA Sophia Antipolis, France. \\ bard@epidaure.inria.fr, na@epidaure.inria.fr \\ ${ }^{2}$ CEREMADE, U.R.A. CNRS 749, Université Paris IX - Dauphine, France. \\ cohen@ceremade.dauphine.fr
}

Corresponding author:

Eric BARDINET

EPIDAURE Project

INRIA Sophia Antipolis

2004 Route des Lucioles, B.P. 93

06902 Sophia Antipolis CEDEX, France

Email: Eric.Bardinet@sophia.inria.fr,

Phone: +339365 7665 Fax: +339365 6669 


\begin{abstract}
In many computer vision and image understanding problems, it is important to find a smooth surface that fits a set of given unstructured 3D data.

Although approaches based on general deformable models give satisfactory results, in particular a local description of the surface, they involve large linear systems to solve when dealing with high resolution $3 \mathrm{D}$ images. The advantage of parametric deformable templates like superquadrics is their small number of parameters to describe a shape. However, the set of shapes described by superquadrics is too limited to approximate precisely complex surfaces. This is why hybrid models have been introduced to refine the initial approximation.

This article introduces a deformable superquadric model based on a superquadric fit followed by a free form deformation (FFD) to fit unstructured 3D points. At the expense of a reasonable number of additional parameters, free form deformations provide a much closer fit and a volumetric deformation field.

We first present the mathematical and algorithmic details of the method. Then, since we are mainly concerned with applications for medical images, we present a medical application consisting in the reconstruction of the left ventricle of the heart from a various number of 3D cardiac images. The extension of the method to track anatomical structures in spatio-temporal images (4D data) will be presented in a companion article [9].
\end{abstract}

KEYWORDS: Surface reconstruction, Deformable models, Parametric models, Superquadrics, 3D Splines, Free-form deformations, Regularization. 


\section{Introduction}

In many computer vision and image understanding problems, it is important to find a smooth surface that fits a set of given unstructured 3D data. In particular, in the large domain of medical image understanding, one often needs to solve fitting problems, in order to make surface reconstruction and get a precise compact representation of anatomical structures.

Over the last twenty years, many fitting problems have been formulated as the minimization of an energy functional corresponding to a model of the surface $[32,14,38,58,23,40,27]$. Although these approaches, based on general deformable surfaces, give satisfactory results, in particular a local description of the surface, they involve large linear systems to solve, in particular when dealing with high resolution $3 \mathrm{D}$ images. This is why we investigate in this article the introduction of adequate parametric models to describe surfaces in 3D images.

The advantage of parametric deformable templates, like superquadrics or hyperquadrics [54, 35], is their small number of parameters to represent a shape. The minimization of the energy associated to the model is done in a reduced space of admissible solutions. Although superquadric shapes give a good global approximation to a surface, the set of shapes described by superquadrics is too limited to approximate precisely complex surfaces. This is why hybrid models have been introduced (see for example $[59,19])$, to refine the initial approximation.

In this article, we introduce a deformable superquadric model based on a superquadric fit followed by a free form deformation (FFD) to fit unstructured 3D data. This model will yield a compact representation of complex 3D objects, allowing important data compression. It will also be possible, 1 due to the volumetric aspect of the FFD, to deform simultaneously several objects. Our model may be used for surface reconstruction and fitting problems involving any kind of images or applications. The only assumption is that we require some knowledge on the global shape of the objects we deal with. Since we are mainly concerned with applications for medical images, precisely for the heart analysis, we present a medical application of our model, consisting in the reconstruction of the left ventricle of the heart. For this application, it is relevant to use superellipsoids as initial shape. For different applications, our model could be easily modified by taking as initial shape some other type of superquadrics or even supertoroids instead of superellipsoids.

The extraction and representation of the left ventricular wall is an important topic, because cardiovascular diseases are still the first cause of mortality in developed countries. Medical image analysis can help the diagnosis of such diseases by assessing a number of quantitative and objective numerical parameters describing the behavior of the cardiac system. Several imaging techniques produce temporal series of three-dimensional description of the heart, i.e. $4 \mathrm{D}(3 \mathrm{D}+\mathrm{T})$ images. Among them, 
gated MRI, ultrasound images and nuclear medicine images; also, the DSR (Dynamic Spatial Reconstructor), a fast CT-scanner developed at the Mayo Clinic, used to produce such 4D images.

Because it is characteristic of the good health of the heart, the left ventricle motion and deformation has been extensively studied by medical image processing groups as well as hospitals. Since its creation in 1989, our group has pioneered work in the use of deformable models to extract the left ventricle $[4,21,5,20,23,6,3,44,13]$. Other groups as well have also made various contributions to understanding the complex deformation of the ventricle $[2,43,1,42,18,52]$.

We use the a priori information that the shape of the left ventricle resembles an ellipsoid deformed by a smooth transformation, and limit strongly the number of parameters necessary for this representation. We also increase the robustness of the fit in the presence of noise or sparse data points. Other approaches for the segmentation of the left ventricle using parametric deformable models include [59, 61, 45].

After a description of the parametric model in Section 2, we explicit the details of our fitting algorithm, both for the superellipsoid model and for the FFD computation (Section 3). We then present two ways of controlling explicitly the regularity of the model (Section 4). Finally we show in Section 5 three experimental studies which demonstrate the effectiveness of this approach to capture the 3D shape of the left ventricle in a various number of 3D images, with a reduced number of parameters. The temporal tracking of these parameters will be described in a companion article [9].

\section{Fitting 3D data with a superquadric}

Superquadrics have been defined by A. Barr [11]. They are an extension of the quadrics family, based on the already old notion of superellipse [30]. In the field of computer graphics and CAD/CAM, this new family of parametric shapes has been widely used. In computer vision, their first use is due to A. Pentland [47], followed by many other authors [7, 16, 53, 28, 29, 48, 54, 33, 41, 34].

As for ordinary quadrics, there are four kinds of superquadrics (provided that one considers torus as a special quadric): superellipsoid, superhyperboloid with one or two sheets, supertorus. In the following, we will only consider the case of superellipsoids. Indeed, the family of surfaces described by superellipsoids is the most adapted for the representation of the anatomical structures in which we are interested. In the case of other applications, the technique presented in this article can be easily adapted to the three other kinds of superquadrics with very similar equations.

We first recall the definition of superellipsoids, then we introduce a new parameterization which gives a uniform distribution of points on the model surface. We will then describe the method to fit $3 \mathrm{D}$ data with these surfaces and give a geometric interpretation of the criterion of fit. 
Additional details are presented in Appendix A.

\subsection{Definition of superquadrics}

Superquadrics form a family of implicit surfaces obtained by extension of usual quadrics. Superellipsoids are defined by the implicit equation:

$$
\left(\left(\left(\frac{x}{a_{1}}\right)^{\frac{2}{\epsilon_{2}}}+\left(\frac{y}{a_{2}}\right)^{\frac{2}{\epsilon_{2}}}\right)^{\frac{\epsilon_{2}}{\epsilon_{1}}}+\left(\frac{z}{a_{3}}\right)^{\frac{2}{\epsilon_{1}}}\right)^{\frac{\epsilon_{1}}{2}}=1 .
$$

The natural parameterization, coming from the spherical one, can be written:
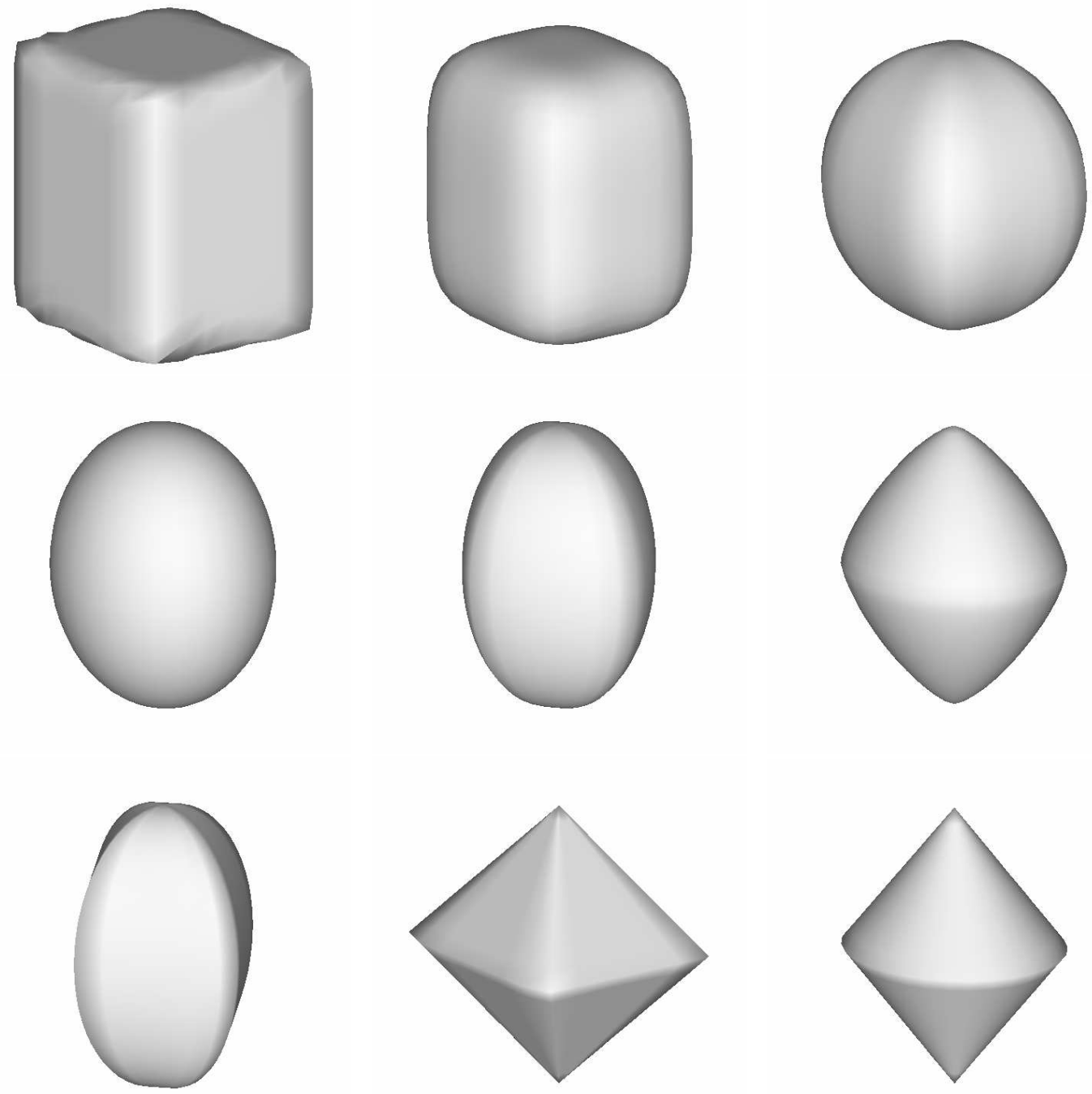

Figure 1: Examples of superellipsoids: a wide variety of shapes. 


$$
\boldsymbol{x}(\eta, \omega)=\left[\begin{array}{l}
a_{1} \cos ^{\epsilon_{1}} \eta \cos ^{\epsilon_{2}} \omega \\
a_{2} \cos ^{\epsilon_{1}} \eta \sin ^{\epsilon_{2}} \omega \\
a_{3} \sin ^{\epsilon_{1}} \eta
\end{array}\right], \quad\left\{\begin{array}{c}
-\pi / 2 \leq \eta \leq \pi / 2 \\
-\pi \leq \omega<\pi
\end{array}\right.
$$

The implicit equation of the superellipsoid permits to define an inside-outside function $F$ :

$$
F(x, y, z)=\left(\left(\left(\frac{x}{a_{1}}\right)^{\frac{2}{\epsilon_{2}}}+\left(\frac{y}{a_{2}}\right)^{\frac{2}{\epsilon_{2}}}\right)^{\frac{\epsilon_{2}}{\epsilon_{1}}}+\left(\frac{z}{a_{3}}\right)^{\frac{2}{\epsilon_{1}}}\right)^{\frac{\epsilon_{1}}{2}} .
$$

Superquadrics are real mathematical solids. Therefore, the values of $F$ define 3 distinct regions of the 3D space:

$$
\left\{\begin{array}{l}
\text { if } F(x, y, z)=1,(x, y, z) \text { is on the surface, } \\
\text { if } F(x, y, z)>1,(x, y, z) \text { is outside, } \\
\text { if } F(x, y, z)<1,(x, y, z) \text { is inside. }
\end{array}\right.
$$

\subsection{Regular parameterization}

The previous parameterization (Eq. (2)) has the advantage, for CAD/CAM applications, to supply more points in large curvature areas (see Figure 2). But, to fit a set of 3D points with a superellipsoid, it is better to have a more uniform distribution of the points on the surface. Vemuri and al. [61] presented a solution to this problem by the resolution of a differential equation. We propose a less expensive solution.

Let us first consider the parameterization of a sphere in spherical coordinates:

$$
\left\{\begin{array}{l}
x=\cos \eta \cos \omega, \quad-\frac{\pi}{2} \leq \eta \leq \frac{\pi}{2} \\
y=\cos \eta \sin \omega, \quad-\pi \leq \omega<\pi \\
z=\sin \eta
\end{array}\right.
$$

By a constant step discretization grid of $\left[-\frac{\pi}{2}, \frac{\pi}{2}\right] \times[-\pi, \pi]$, the points on the sphere are regularly positioned. It is then enough to project a ray from the center through each of these points of the sphere and find the intersection with the superellipsoid to get a more uniform distribution of the points on it.

This is done in two steps:

- From the sphere to the ellipsoid:

$$
x_{e}=a_{1} x \quad ; \quad y_{e}=a_{2} y \quad ; \quad z_{e}=a_{3} z
$$

- From the ellipsoid to the superellipsoid:

$$
x_{s}=\rho x_{e} \quad ; \quad y_{s}=\rho y_{e} \quad ; \quad z_{s}=\rho z_{e}
$$



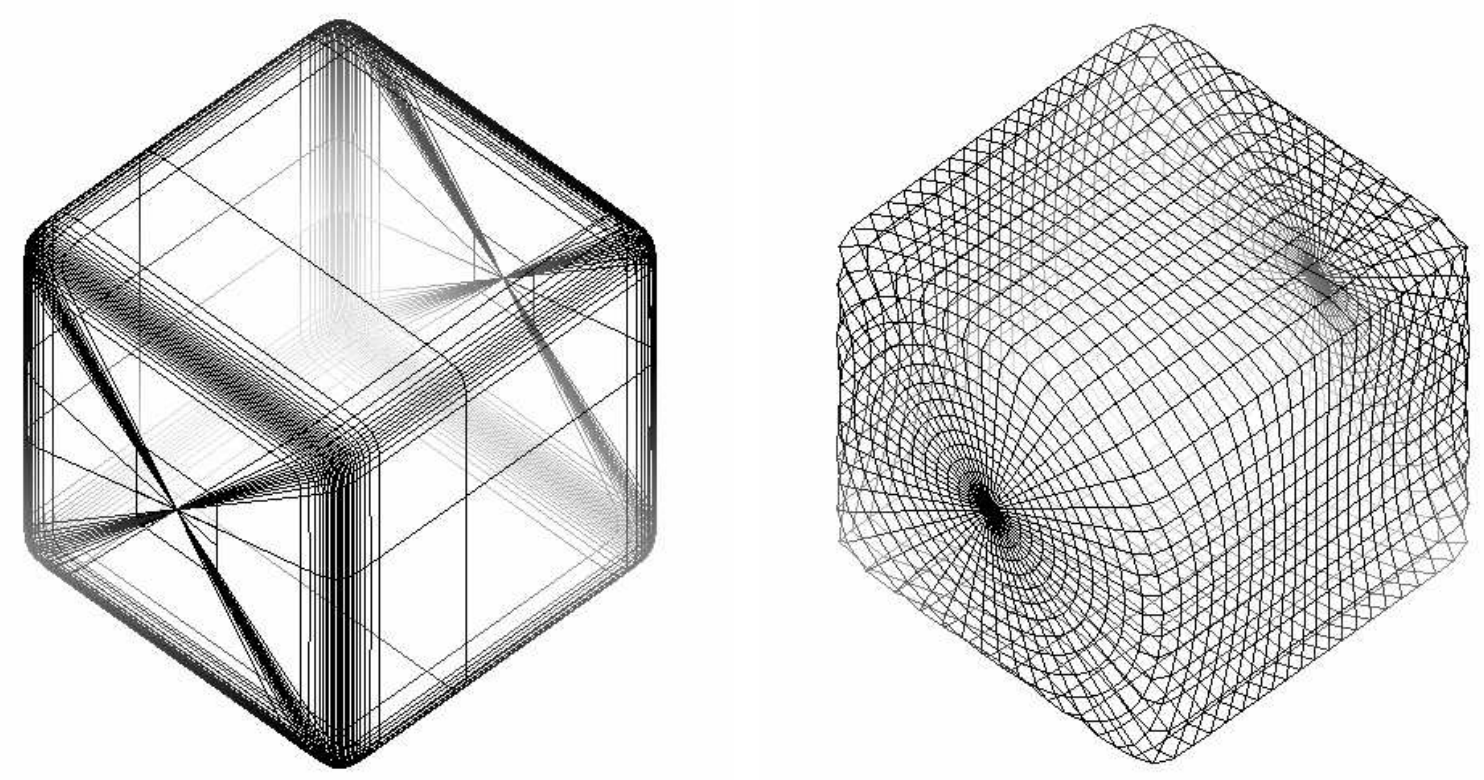

Figure 2: Left: curvature-dependent parameterization. Right: uniform parameterization.

Replacing $x, y$ and $z$ by $x_{s}, y_{s}$ and $z_{s}$, in Eq. 1, we obtain an expression of $\rho$ as a function of $\eta$ and $\omega$ :

$$
\rho=\left[\left(|\cos \omega \cos \eta|^{\frac{2}{\epsilon_{2}}}+|\sin \omega \cos \eta|^{\frac{2}{\epsilon_{2}}}\right)^{\frac{\epsilon_{2}}{\epsilon_{1}}}+|\sin \eta|^{\frac{2}{\epsilon_{1}}}\right]^{-\frac{\epsilon_{1}}{2}}
$$

We then obtain a new parameterization of the superellipsoid by the computation of $\rho, x_{s}, y_{s}$ and $z_{s}$ for each value of the grid on the domain $\left[-\frac{\pi}{2}, \frac{\pi}{2}\right] \times[-\pi, \pi]$. The points are then regularly spaced on the surface (see Figure 2). Remark that the computation is more costly than for the natural parameterization: 2 additions, 3 multiplications and 1 power for each point. However we now consider this parameterization of superellipsoids, to get a more uniform distribution of the points on the surface.

\section{$2.3 \quad$ Fit}

The use of superquadrics for the analysis of scenes in computer vision was first introduced by Pentland [47]. He proposed a heuristic approach based on a search over the whole parameter space for the best value of the goodness-of-fit criteria. Since this approach is computationally very expensive, later authors prefered iterative algorithms, in particular, Solina and Bajcsy have used superellipsoids for the approximation of 3 -D objects $[7,53,54]$. Our fitting algorithm is inspired by their work. 


\section{Least squares minimization:}

Suppose that the data that we want to fit with the superellipsoid are a set of $3 \mathrm{D}$ points $\left(x_{d}, y_{d}, z_{d}\right), i=1, \ldots, N$. The goal of the algorithm is to change the eleven parameters to find the values for which most of the $3 \mathrm{D}$ points are close to the surface of the model. In the general case, there will not exist a set of parameters for which the model perfectly fits to the data, because the shapes which we are interested in are more complex than superellipsoids. Therefore, the problem has to be formulated as a least-squares problem. Now, a point on the surface of the superellipsoid satisfies $\widehat{F}=1$ (this function corresponds to the inside-outside function $F$ after a rigid transform, see Appendix A), therefore we can search for the minimum of the following energy:

$$
E(A)=\sum_{i=1}^{N}\left[1-\widehat{F}\left(x_{d}, y_{d}, z_{d}, a_{1}, a_{2}, a_{3}, \epsilon_{1}, \epsilon_{2}, \varphi, \theta, \psi, \boldsymbol{t}\right)\right]^{2} .
$$

The derivatives of $E$ with respect to the eleven parameters of the superellipsoid can be explicitly computed. The minimum of the energy is computed by using the algorithm of multidimensional conjugate gradient method [50]. This method localizes the minimum of a function only with the computation of its first derivatives, and insures a fast convergence. Figure 3 represents the result
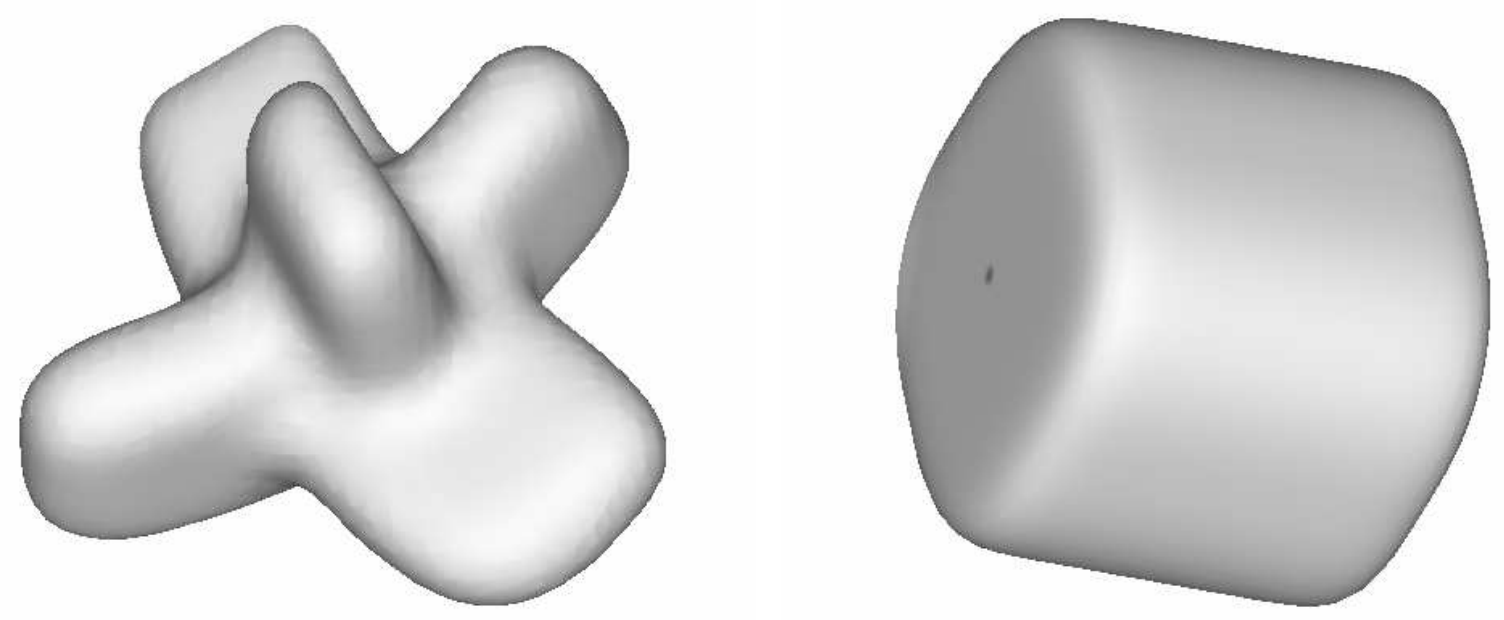

Figure 3: Left: Synthetic 3D data. Right: Fit with a superellipsoid.

of the fit of a superellipsoid on a synthetic set of 3D points (three crossing superquadrics deformed by a global tapering, see [12]). One can see that, if the superellipsoid provides a rough estimate of 
the shape, the model clearly has to be refined, as we will see in the next section. However, let us point out that if the set of $3 \mathrm{D}$ points is closer to a superellipsoid shape, then the result is as we may expect (see figure 7).

\subsection{Geometric interpretation}

The exact computation of the Euclidean distance between a 3D point and a surface is very expensive and usually an approximation is computed by use of iterative filters $[15,26]$. In this section, we detail two analytic expressions of the approximation of the distance between a point and an implicit surface. The first one provides a geometric interpretation of the least-squares criterion which is used to fit the data with a superellipsoid.

- Intrinsic approximation:

Let us consider a superellipsoid in its intrinsic local frame (Eqs. (2) and (1)). Let $M=(x, y, z)$ be a data point. The line joining the center $O$ of the local frame and $M$ intersects the superellipsoid surface at $M_{0}=\left(x_{M_{0}}, y_{M_{0}}, z_{M_{0}}\right)$ (see figure 4). We want to calculate the distance $\left\|M_{0} M\right\|$. Since $O, M_{0}$ and $M$ are on a same line, we can write : $\overrightarrow{O M_{0}}=\mu \overrightarrow{O M}$ with $\mu>0$. Therefore we have $\left(x_{M_{0}}, y_{M_{0}}, z_{M_{0}}\right)=(\mu x, \mu y, \mu z)$, and since $F(\mu x, \mu y, \mu z)=$ $\mu F(x, y, z)$, with $F\left(M_{0}\right)=1$, we obtain the value of $\mu$ :

$$
\mu=\frac{1}{F(x, y, z)}
$$

We then deduce the following:

$$
\left\{\begin{aligned}
\left\|M_{0} M\right\| & =|1-\mu|\|O M\| \\
& =\left|\frac{F(x, y, z)-1}{F(x, y, z)}\right|\|O M\| \\
\left\|M_{0} M\right\| & =\left|\frac{1-\mu}{\mu}\right|\left\|O M_{0}\right\| \\
& =|F(x, y, z)-1|\left\|O M_{0}\right\|
\end{aligned}\right.
$$

And:

$$
(F-1)^{2}=\left(\frac{\left\|M_{0} M\right\|}{\left\|O M_{0}\right\|}\right)^{2}=\left(\frac{\|O M\|}{\left\|O M_{0}\right\|}-1\right)^{2} .
$$

This gives a geometric interpretation of the least-squares criteria as an intrinsic homothetic characteristic. This approximation is always greater than the exact Euclidean distance $d=\|$ $M P \|$, as one can see in Figure 4.

- First order approximation:

We also have an approximation of $d$ when the data point $M$ is close to its projection $P$ on 
the superellipsoid.

$$
d \approx \frac{|F(M)-1|}{\|\nabla F(M)\|}
$$

It is also possible to define a more precise approximation that is bounded as shows Taubin [57], but it is computationally more expensive.
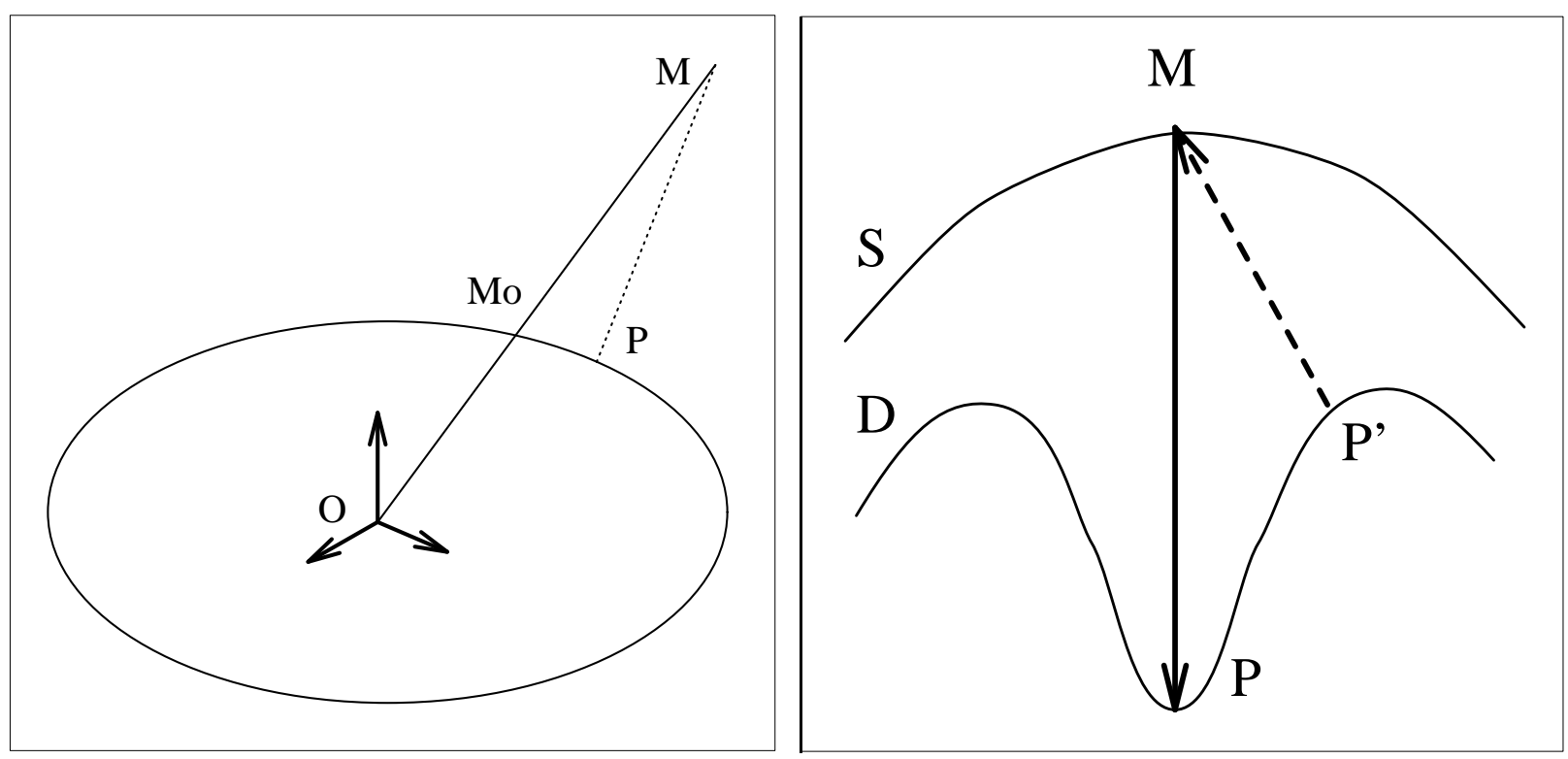

Figure 4: Left: Approximation of the Euclidean distance between a point and the superellipsoid. Right: Two ways to compute the closest data point to $M$.

\section{Refinement of the fit with free form deformations}

After the fit of a superellipsoid, we have a parametric representation of the 3D data. The model is correctly oriented with respect to axes of inertia of the data, and the three axes already have correct dimensions. But the model is not close enough to the data, because the set of shapes described by superellipsoids is too limited (mainly because of the symmetry of superellipsoids, as well as other kinds of superquadrics) for the description of complex structures such as the anatomical ones, e.g. the cardiac left ventricle.

Thus we have to refine this representation. As we mentioned in the introduction, several hybrid referee models, combining a superquadric model with local deformations, have already been proposed. 1 Among them, Sclaroff and Pentland [49] define a local normal displacement, Vemuri and Radisavljevic [60] add local deformations decomposed in a wavelet basis and Terzopoulos and Metaxas [59] 
describe the problem from a mechanical point of view, defining a dynamic elastic model where the local deformations are expressed like surfacic spline functions. Let us point out that these models are again local models, with a global shape memory.

To refine the superellipsoid fit, we will combine it with a global volumetric deformation called free form deformation (FFD). It is a tool devoted to the deformation of solid geometric models in a free-form manner. FFDs can also deform all kinds of surfacic primitives: planes, implicit surfaces, quadrics, superquadrics.

Using FFDs yields one major advantage. The resulting deformable superellipsoid is defined by a referee reduced number of parameters, instead of the position of every model point. This typical feature will 1 allow us to represent complex 3D data sets by parametric models defined by only a few parameters. Also, because the FFD is a volumetric deformation, several objects can be deformed simultaneously. In the next three sections, we explicit the definition of FFDs, we explain how to use them to refine the superellipsoid representation by solving an inverse problem and we finally present an iterative two-step algorithm.

\subsection{Definition of free form deformations}

FFDs were introduced by Segerberg and Parry [51] in computer graphics. An analogy, to understand FFDs, is to consider a rubber-like box in which the object that we want to deform is embedded. To deform the object, one just needs to deform the box, and the object follows in an way similar to an elastic parallelepiped the volumetric deformation of the box. FFDs became very popular in the field of CAD/CAM, essentially because the object deforms in a very intuitive way with respect to the deformation transmitted to the box.

More precisely, FFDs are an application from $\mathbb{R}^{3}$ to $\mathbb{R}^{3}$, defined by the tensor product of trivariate Bernstein polynomials. The use of $\mathbb{R}^{3}$ to $\mathbb{R}^{3}$ applications already appears in a paper from A. Barr [12] on specific regular deformations, namely for twisting, bending and tapering geometric solids. The principle of FFDs is as follows: the object to be deformed is embedded in a 3D box. Inside this box, a volumetric grid of points is defined, which links the box to the object (by the previously defined polynomial which defines the deformation function). The box is then deformed by the displacement of its lattice, and the deformation is transmitted to the object with the deformation function (see Figure 5).

Several extensions of FFDs were published. In particular, Coquillart [24, 25] replaced the trivariate Bernstein polynomials (associated to Bezier curves) by cubic B-splines to define the deformation functions, and mainly expanded the notion of parallelepipedic box to free form grids of control points; that permits to be closer to the object to be deformed. Let us quote also Griessmair et al. 

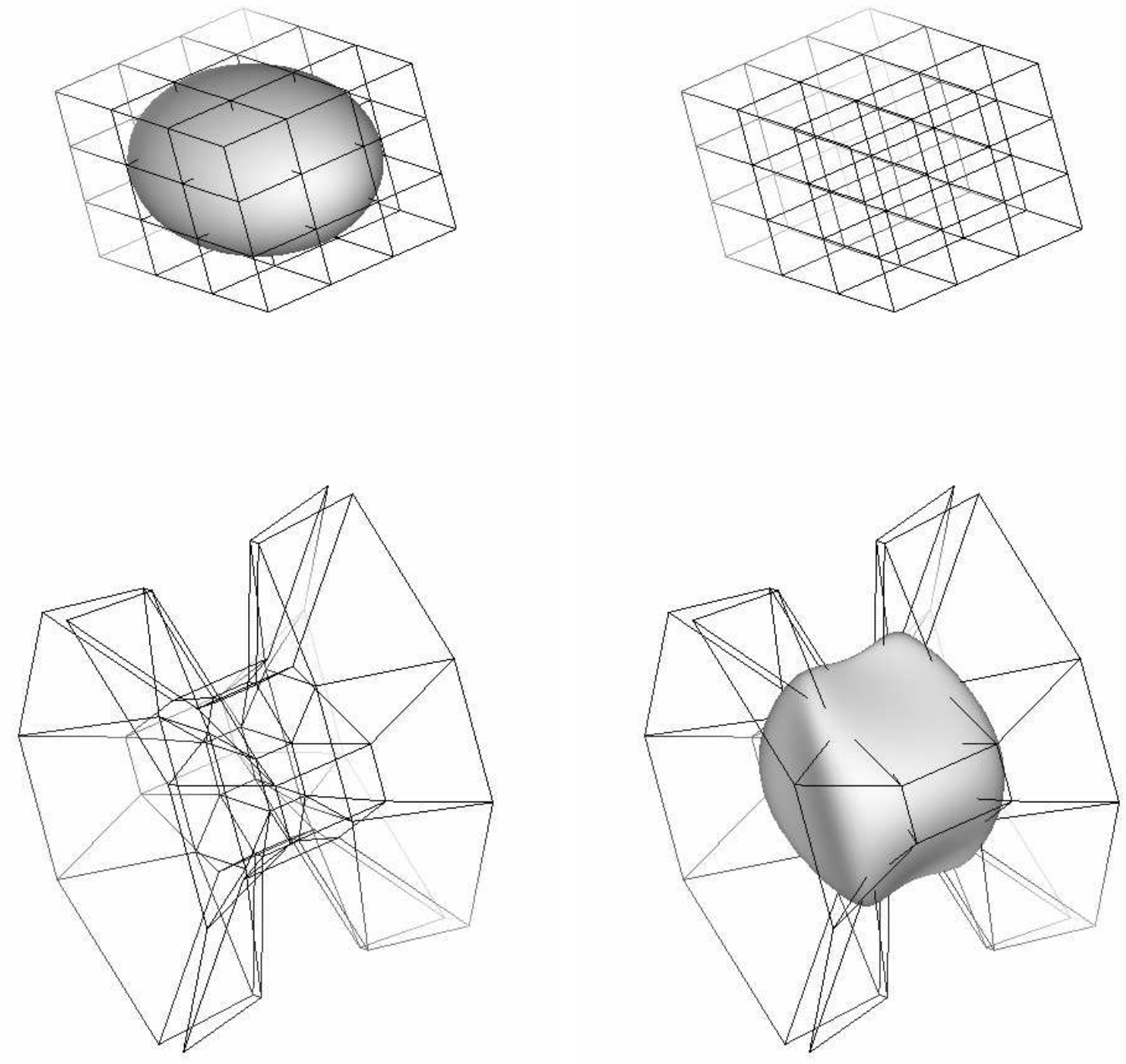

Figure 5: Free form deformations in computer graphics. Top left: object embedded in the initial box of control points. Top right: initial box. Bottom left: deformed box. Bottom right: resulting deformed object in the deformed box. 
[31], Chang et al. [17], who propose a generalized De Casteljau algorithm to compute the model deformed by an FFD, and Lamousin et al. [39] who use NURBS (non uniform rationnal B-splines) as deformation functions.

We decided to use the original definition of FFDs [51]. The motivation of this choice is essentially practical. Actually, the use of Bernstein polynomials as deformation functions, and the use of parallelepipedic boxes to define grids of control points insures a fast computation of FFDs and gives good results for the representation of medical 3D data.

The FFD formulation is divided into two steps:

1. Computation of the local coordinates of the object points in the frame defined by the box of control points.

2. Displacement of the control points and estimation of the new position of the deformed object.

The corresponding equations are presented in Appendix B. An important characteristic of FFDs is that a parametric surface remains parametric after deformation. A complete description of FFDs can be found in [51] and [46]. Figure 5 is an illustration of FFDs.

\subsection{Solving the inverse problem}

The superellipsoid fit provides a first parametric approximation of the set of 3D data, which is often a crude approximation of the original data points set. We want to use FFDs to refine the representation of the data we obtained with the superellipsoid. The main interest of FFDs is that the resulting deformation of the object is just defined by the position of the deformed box, instead of the displacement of every model point. This typical feature will allow us to represent complex $3 \mathrm{D}$ data sets by a few parameters, and we will be able to reach compression ratios of the order of 50.

Solving the inverse problem will allow us to deform the superellipsoid with respect to the data. Hsu et al [37] deal with a simplified version of this problem in the field of CAD/CAM. Their problem was to be able to directly manipulate points of a model deformed by FFDs.

\subsubsection{Computation of the deformation function}

First, we need to define a parallelepipedic box which contains the original superellipsoid. To estimate the size and orientation of this box, we use 6 of the 11 parameters which characterize the superellipsoid. The sizes $a_{1}, a_{2}, a_{3}$ according to the 3 axes of inertia define the size of the box, and its orientation is given by the coefficients of the rigid transform $\varphi, \theta, \psi, t_{x}, t_{y}, t_{z}$ of the superellipsoid. 
We then create the volumetric grid of $(l+1)(m+1)(n+1)$ control points in the box. Eq. (17) can be rewritten:

$$
\left\{\begin{aligned}
x\left(\boldsymbol{P}_{\boldsymbol{i} \boldsymbol{k} \boldsymbol{k}}\right) & =a_{1}\left(1-2 \frac{i}{l}\right), \\
y\left(\boldsymbol{P}_{\boldsymbol{i} \boldsymbol{k} \boldsymbol{k}}\right) & a_{2}\left(-1+2 \frac{j}{m}\right), \\
z\left(\boldsymbol{P}_{\boldsymbol{i j k}}\right) & =a_{3}\left(1-2 \frac{k}{n}\right),
\end{aligned}\right.
$$

if we choose as the local frame origin, the point $X_{0}$ with coordinates $\left(a_{1},-a_{2}, a_{3}\right)$. The application of the rigid transform finally leads to the correct box.

Eq. (18) of Appendix B, which links control points $\boldsymbol{P}_{\boldsymbol{i j} \boldsymbol{k}}$ to model points $\boldsymbol{X}$, can be written in a matrix form:

$$
\boldsymbol{X}=\boldsymbol{B P},
$$

where $\boldsymbol{B}$ is the deformation matrix $N D \times N P(N D$ : number of points on the superellipsoid, $N P$ : number of control points $=(l+1)(m+1)(n+1)), \boldsymbol{P}$ is a matrix $N P \times 3$ which contains coordinates of the control points $\boldsymbol{P}_{\boldsymbol{i j k}}$, and $\boldsymbol{X}$ a matrix $N D \times 3$ with coordinates of the model points.

The grid of control points being defined, it is sufficient to compute the local coordinates of the superellipsoid points to get the deformation matrix $\boldsymbol{B}$.

Actually Eq. (5) corresponds to three separate linear systems, one for each coordinate. Since the matrix of the three systems is the same, we will from now on consider that this equation stands for the first coordinate of points ( $\boldsymbol{X}$ and $\boldsymbol{P}$ column vectors).

\subsubsection{Evaluation of a displacement field between the superellipsoid and the data}

We need to evaluate a displacement field between the superellipsoid and the data (we suppose again it is a set of 3D points). Figure 4 represents an oversimplified section of the approximation of data $D$ by a superellipsoid $S$. To point $M$, we want to associate its closest point on $D$. There are two candidates, $P$ and $P^{\prime}$. To get the best approximation of the data $D$, it is better to associate $M$ to $P$. This choice is still valid for other kinds of data, in the sense that data will always have a more complex shape than their approximation by a superellipsoid.

A usual technique consists in the computation of the data distance map, and then, for each superellipsoid point, search its closest point on $D$. In this case, all the displacement vectors will be directed towards the normal vectors to the data. Thus $M$ will be associated to $P^{\prime}$.

On the other hand, if we compute the distance map of the superellipsoid, and, for each data point, search its closest point on $S$, then the displacement vectors will be directed towards the normal vectors to $S$, and $M$ associated to $P$. But some points of the superellipsoid may not be assigned to a closest point. In that case, a displacement vector is computed by interpolation.

The distance map is computed using an algorithm based on KD-trees (see for example [62]). 

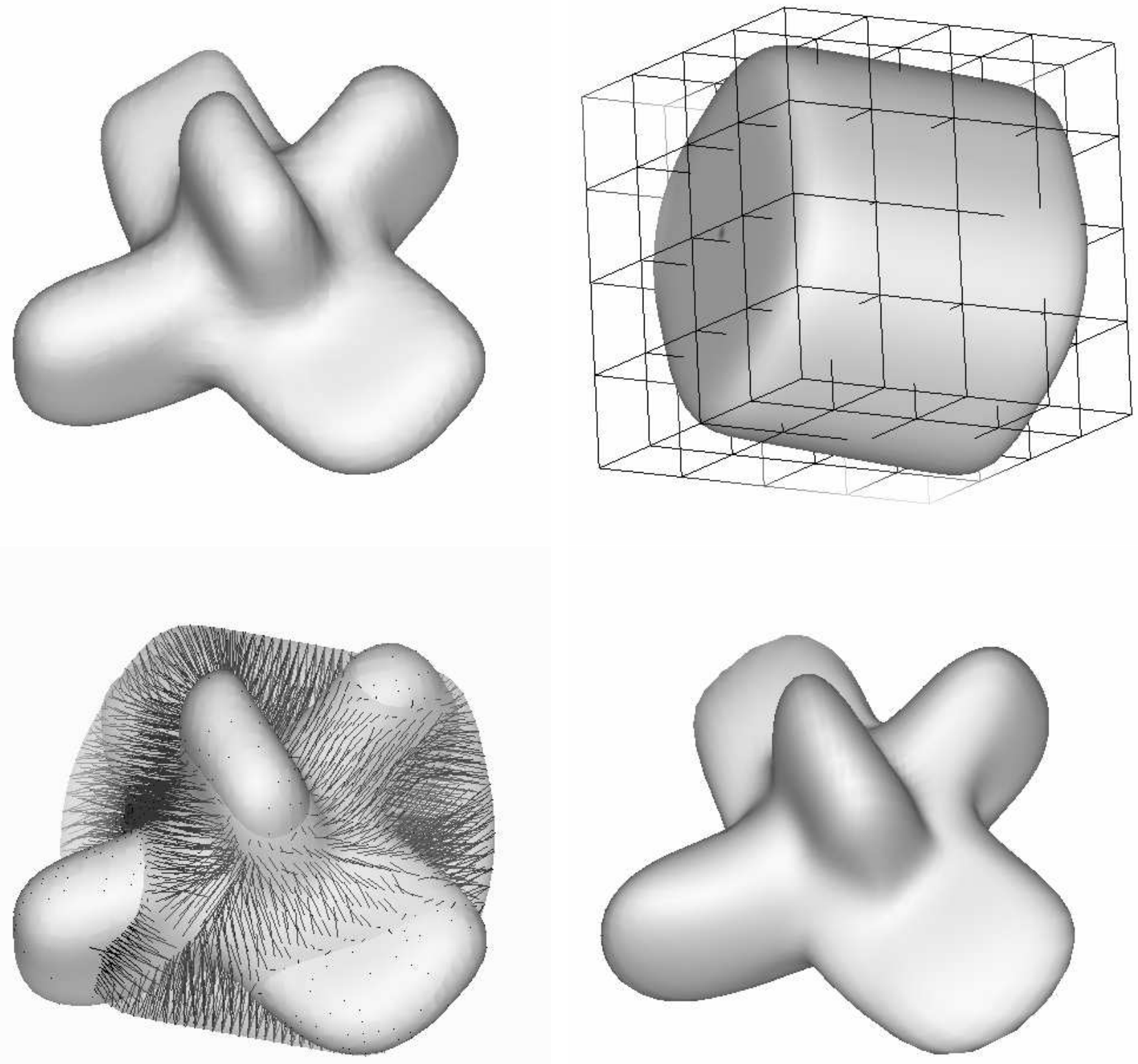

Figure 6: From the superellipsoid to the final model - synthetic example. Top left: data. Top right: superellipsoid fit and initial box of control points. Bottom left: displacement field between data and the superellipsoid. Bottom right: final model after minimization of the displacement field. 

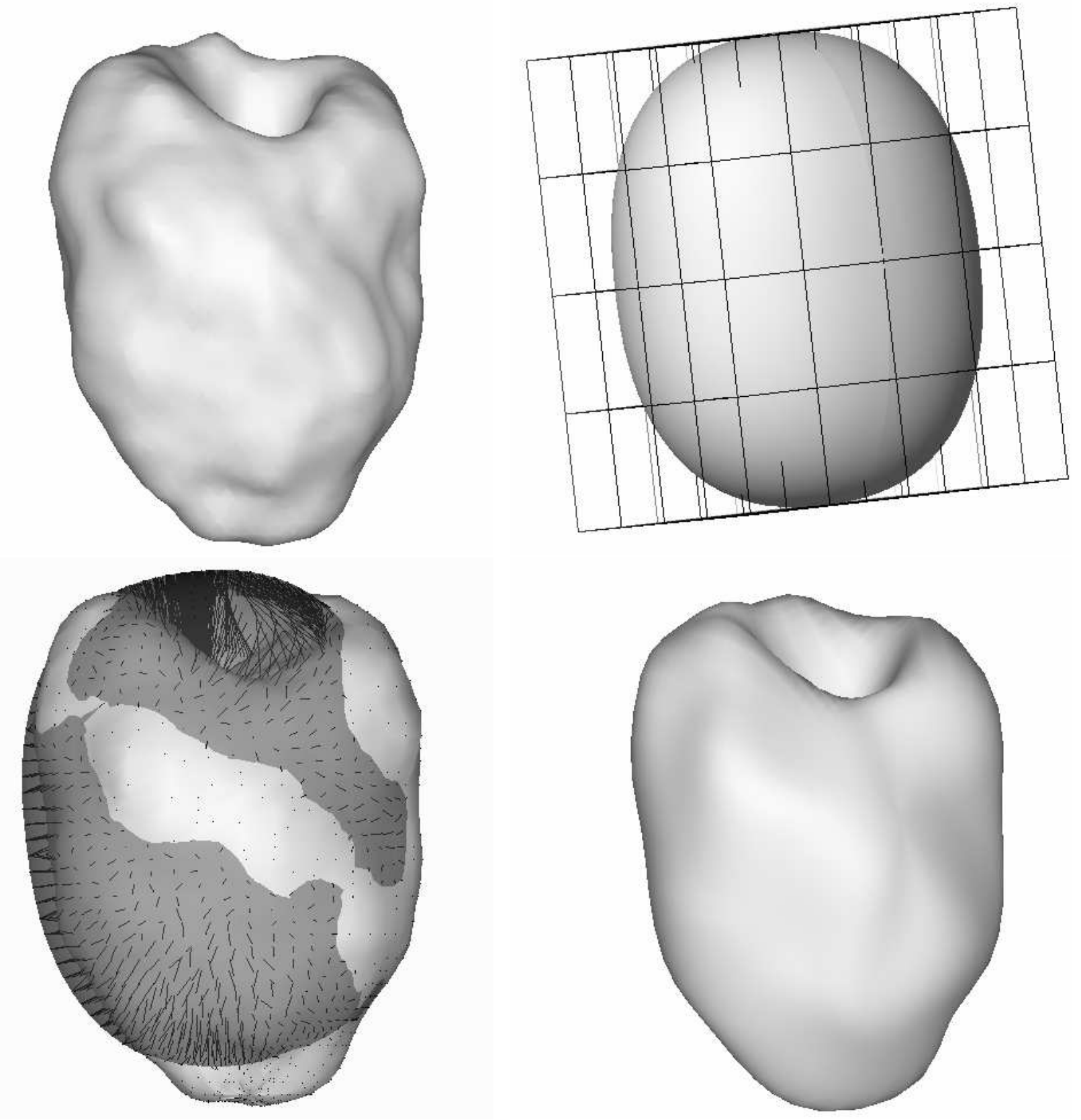

Figure 7: From the superellipsoid to the final model - medical example Top left: data. Top right: superellipsoid fit and initial box of control points. Bottom left: displacement field between data and the superellipsoid. Bottom right: final model after minimization of the displacement field. 


\subsubsection{Deformation of the box of control points}

We now want to deform the superellipsoid toward the data, by deforming the box of control points. If we write $\delta \boldsymbol{X}$ for the displacement field previously computed, the problem we have to solve is equivalent to the resolution of the linear system $(\boldsymbol{X}+\delta \boldsymbol{X})=\boldsymbol{B}(\boldsymbol{P}+\delta \boldsymbol{P})$, which means, using Eq. (5):

$$
\delta \boldsymbol{X}=\boldsymbol{B} \delta \boldsymbol{P}
$$

where $\delta \boldsymbol{P}$ is the variation of the position of control points.

As we said at the beginning of section 3, our goal is to represent data of important size by a parametric deformable model that will be compactly described. This is why the linear system (6) will always be over-determined: the number $N D$ of superellipsoid points is always much larger than the number $N P$ of control points. Therefore there is no exact solution for this problem. It is nevertheless possible to find the best solution in a least-squares sense. So we want to solve the problem:

$$
\min _{\boldsymbol{P}}\|\boldsymbol{B} \boldsymbol{P}-\boldsymbol{X}\|^{2} \equiv \min _{\delta \boldsymbol{P}}\|\boldsymbol{B} \delta \boldsymbol{P}-\delta \boldsymbol{X}\|^{2}
$$

We use a minimization algorithm based on the Singular Value Decomposition (SVD) of matrix $\boldsymbol{B}$.

\subsection{Iterative two-step algorithm}

The resolution of problem (7) leads to a new box of control points, and therefore, by the application of the deformation matrix (Eq. (5)), to a representation model of the data which is more accurate than the initial superellipsoid. Then one can find again a displacement field between this model and the data, and solve again problem (7). Finally, to represent 3D data with a parametric deformable model, we use an iterative two-step algorithm:

- Let $\boldsymbol{P}_{0}$ be the parallelepipedic box of control points; $\boldsymbol{X}_{0}=\boldsymbol{B} \boldsymbol{P}_{0}$ represents the set of points of the initial superellipsoid.

- We iterate the following two steps:

Step 1: Computation of the displacement field $\delta \boldsymbol{X}_{n}$ such as: $\boldsymbol{X}_{n}^{a}=\boldsymbol{X}_{n}+\delta \boldsymbol{X}_{n}$

Step 2: Computation of the control points $\boldsymbol{P}_{n+1}$ by minimization of $\left\|\boldsymbol{B} \boldsymbol{P}-\boldsymbol{X}_{n}^{a}\right\|^{2}$

Computation of the deformed model: $\boldsymbol{X}_{n+1}=\boldsymbol{B} \boldsymbol{P}_{n+1}$

Stop test: computation of the least-squares error $\left\|\boldsymbol{X}_{n+1}-\boldsymbol{X}_{n}\right\|$ 
This algorithm is similar to the formulation of the B-splines snakes with auxiliary variables (see [22]). An essential feature of this approach is that we use a volumetric deformation to deform a surface shape.

Figure 6 (left block) is a detailed example of the complete algorithm applied on synthetic data. Figure 8 represents the associated displacement fields between the model and the data, before and after FFDs. Figure 6 (right block) is a detailed example of the complete algorithm applied to
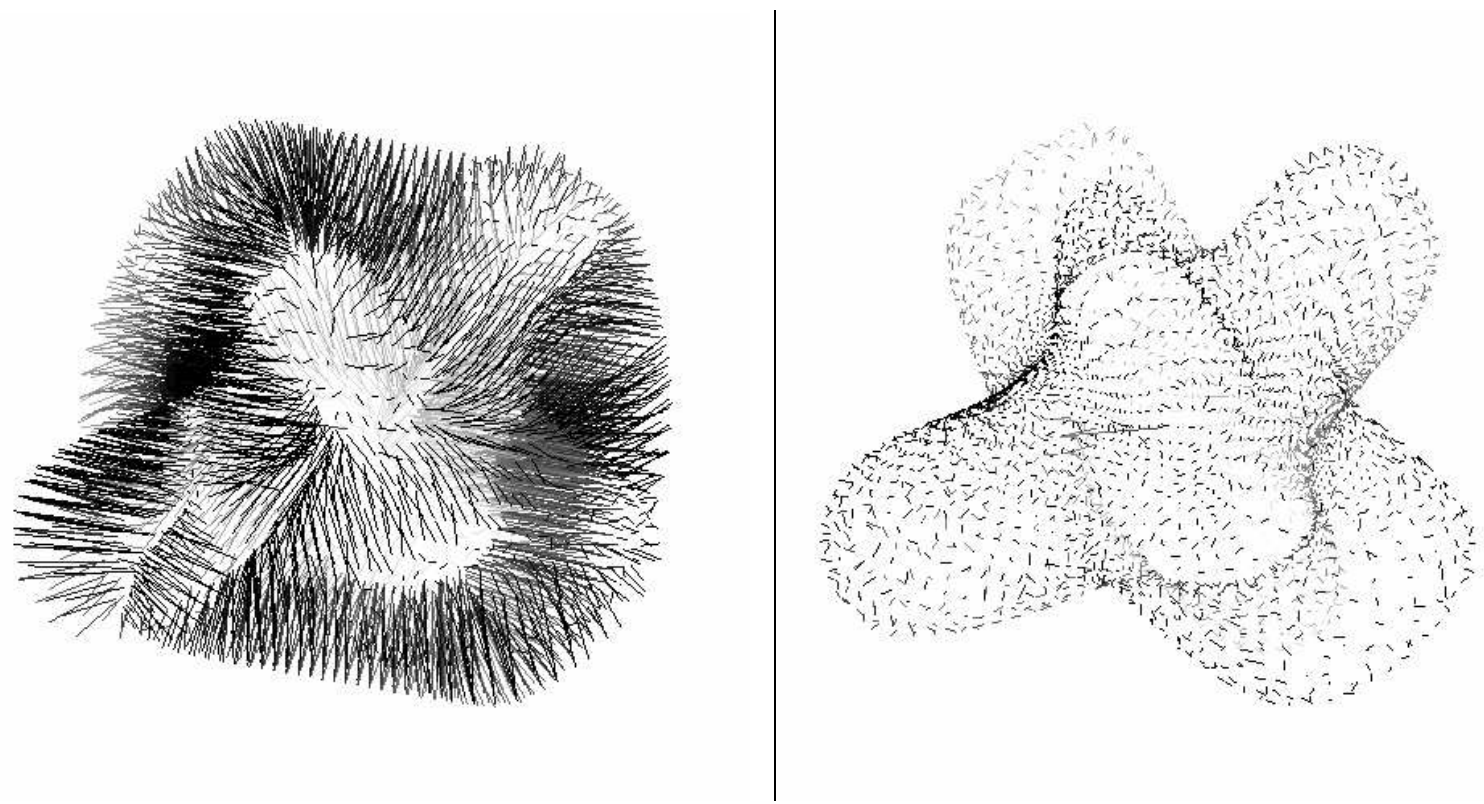

Figure 8: Displacement field of the synthetic example. Left: between the superellipsoid and the data. Right: between the final model (box of size $5 \times 5 \times 5$ ) and the data.

medical data. Remark that the final model is totally defined by the following parameters:

- 11 parameters to describe the superellipsoid fitted to the 3D data.

- $N P$ control points to describe the volumetric deformation applied to the initial model.

From a practical point of view, the number of data points varies between 5.000 and 20.000 , and we use boxes of size $5 \times 5 \times 5$, which means 125 control points. So the representation of those data by the model allows a compression ratio between 40 and 150 . 


\subsection{Particular features of the model}

\subsubsection{Reconstruction from sparse data}

Using a parametric model to represent 3D data permits to increase the robustness of the fit in the presence of sparse data. Figure 9 show the ability of the model to recover a shape using only sparse data. The first example (top row) was generated by intersection of the data showed in Figure 6 (right block) with three orthogonal planes (600 points vs. 6000 points). This is an image that could have been generated by three ultrasound images with different directions. The second example (bottom row) was generated by a uniform subsampling of the same data (900 points vs. 6000 points). This uniform grid of sparse 3D points could be issued from tagged MRI. The shape constraint of the model enables to reconstruct a complete surface which is similar to the model computed from the complete data. With a model defined by a small number of global parameters, there is a strong global shape regularity, contrary to general deformable models, which are defined locally.

\subsubsection{Simultaneous deformation of two surfaces}

An essential feature of this algorithm is that a FFD is a volumetric deformation. This means that several objects can be deformed simultaneously with only one FFD. With two surfaces, for example, using only one model means that the two surfaces are put in a same box of control points, and the minimization of equation (7) is done simultaneously based on the union of the two displacement fields. Using two FFDs for the two surfaces leads to a better quality of approximation. On the other hand, using only one FFD allows to divide the number of parameters by two, yielding a larger compression of the information needed for the description of the parametric model. It also permits to infer from this single FFD, a deformation field over the entire space, due to the volumetric formulation of FFDs (see $[8,10])$.

\section{Regularization of the model}

As shown in the upper left image of figure 10, the control points box may be very irregular. So it becomes difficult to infer quantitative informations on the model deformation from the configuration of those control points. It is the reason why we introduce two possible approachs to explicitly control the regularity of the deformation:

1. Control the norm of the control points displacement with the Singular Value Decomposition of the deformation matrix $\boldsymbol{B}$. 

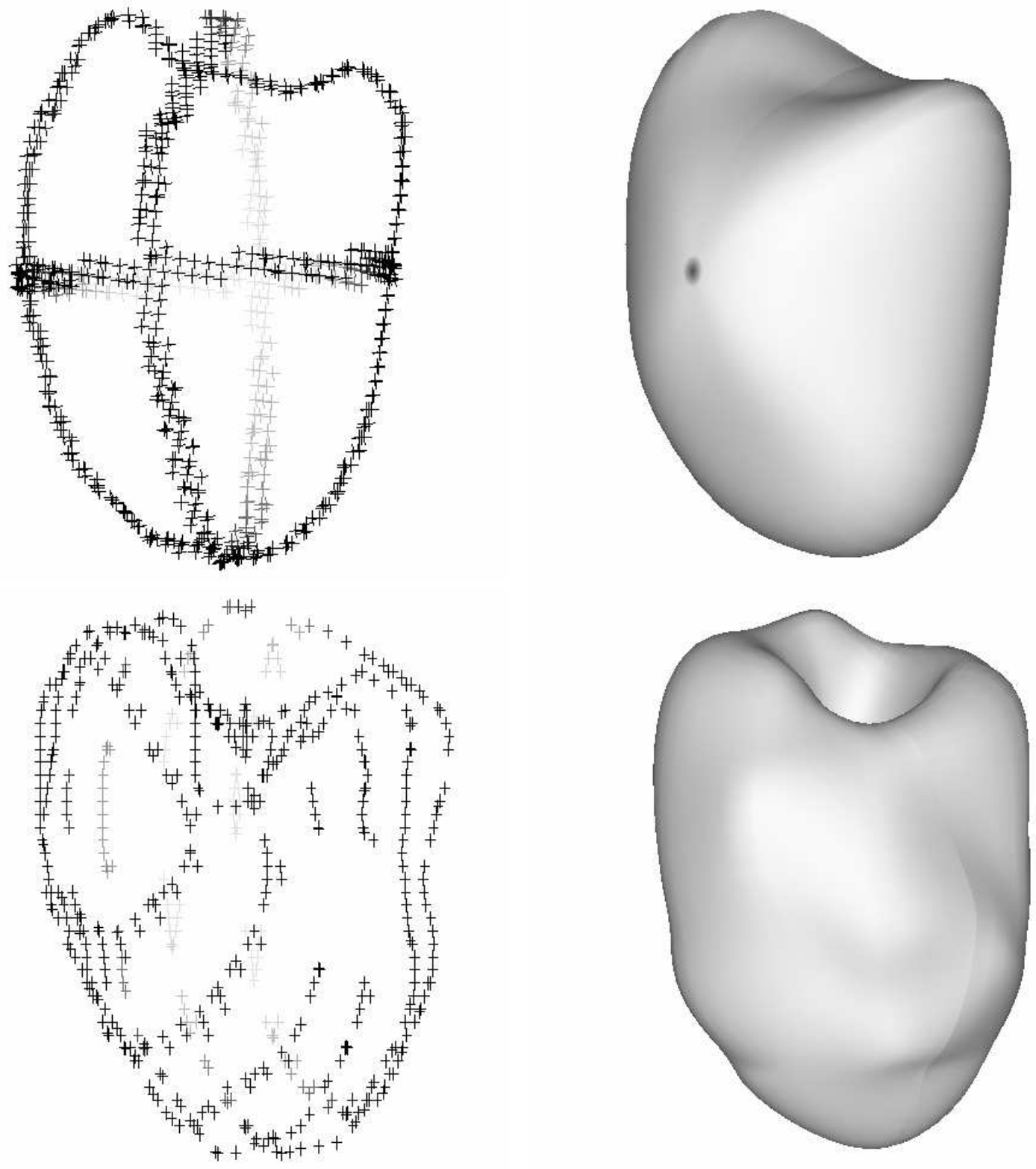

Figure 9: Reconstruction from sparse data. Top row: from three orthogonal planes. Bottom row: uniform subsampling of 6 . Left: data. Right: final model (box of size $5 \times 5 \times 5$ ). 
2. Add a regularizing term in the least-squares criterion (7).

FFDs have also been used to solve some matching problems by Szeliski and Lavallee [55, 56]. To impose a priori regularity constraints on the deformation parameters, they use $m$-order stabilizers.

\subsection{Using the singular value decomposition}

The linear system (Eq. (5)) is solved using Singular Value Decomposition [50]. This decomposition can be written:

$$
\boldsymbol{B}=\boldsymbol{U} \cdot \boldsymbol{W} \cdot \boldsymbol{V}^{T},
$$

where $\boldsymbol{U}$ and $\boldsymbol{V}$ are orthogonal matrix, and $\boldsymbol{W}$ is diagonal. It can be shown that matrix $\boldsymbol{V} \cdot \boldsymbol{W}^{-1} \cdot \boldsymbol{U}^{T}$ is the pseudoinverse of $\boldsymbol{B}$. The elements of $\boldsymbol{W}$ are the nonvanishing singular values of $\boldsymbol{B}$.

By computing the pseudoinverse of $\boldsymbol{B}$ using only the largest singular values (the largest elements of $\boldsymbol{W})$, we control the norm of the solution of (5). Results of this control are shown in figure 10 with a $5 \times 5 \times 5$ box. Figure 11 shows the effect of this control on the approximation accuracy.

\subsection{Using a regularizing term}

By adding a regularizing term, the minimization criterion becomes:

$$
\left\|B P-X_{n}^{a}\right\|^{2}+\alpha \sum_{j=1}^{N P} \sum_{j^{\prime}}\left\|P_{j}-P_{j^{\prime}}\right\|^{2},
$$

where $j^{\prime}$ corresponds to the neighbours of $\boldsymbol{P}$. The second term is an internal energy corresponding to the insertion of zero-length springs between control points. This has a regularizing effect on the box to an extent controlled by the weight $\alpha$. This regularization term can be also written $\|D P\|^{2}$ where $D$ is a matrix which represents a discretized derivative of the control points position.

Figure 12 represents the compared effects of the two regularizations. More precisely, we represent for each of the two methods the correlation between the least-squares approximation error and the norm of the control points box deformation. This figure shows that the two regularization methods are equivalent. 

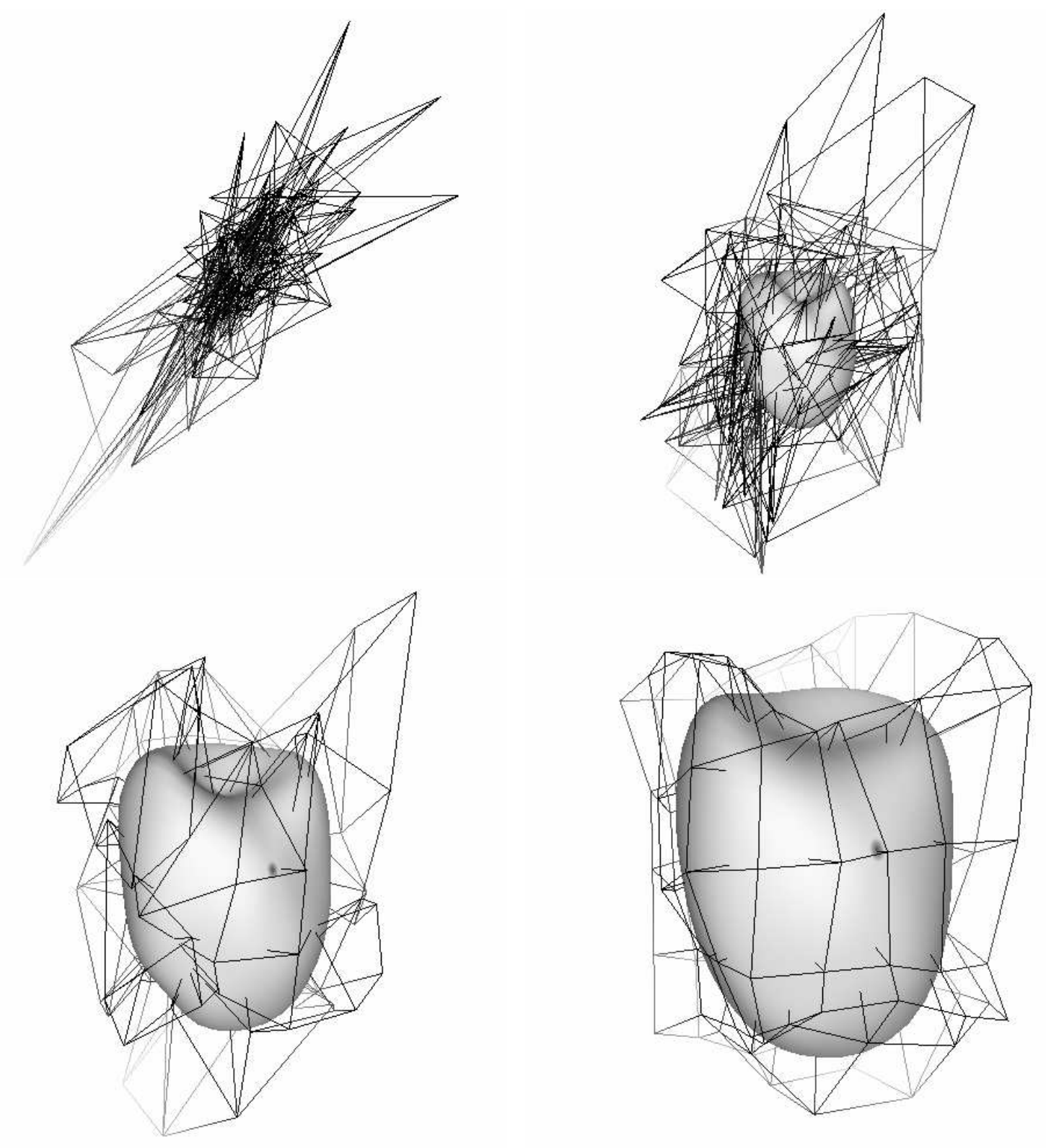

Figure 10: Regularization with SVD (from left to right and top to bottom, the number of diagonal terms is decreasing). The model size changes between the examples are due only to rescaling, the real size is the same). 

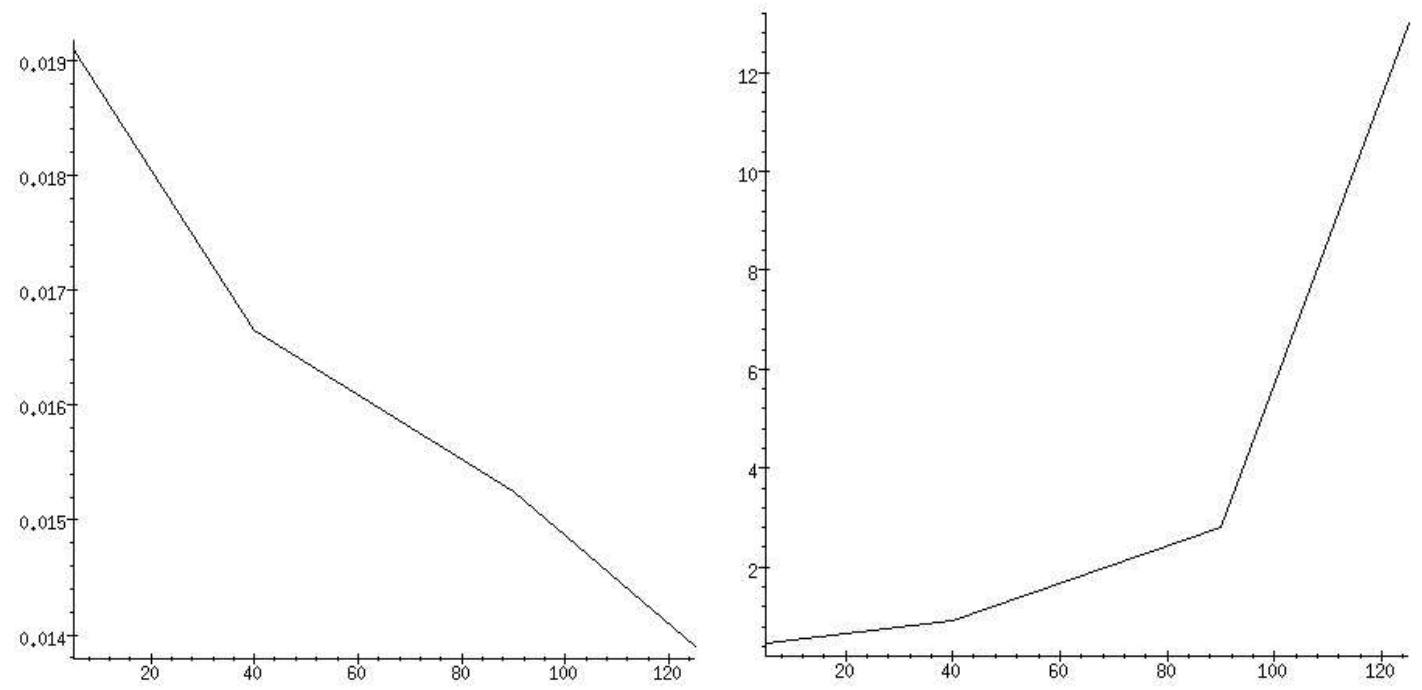

Figure 11: Left: Approximation error between the models and the data (in mm), with a decreasing number of diagonal terms. Right: Deformation of the box of control points (in mm), with a decreasing number of diagonal terms. Note that the 2 curves are made of 4 points and that the index in the horizontal scale corresponds to the 4 examples of Fig. 10.

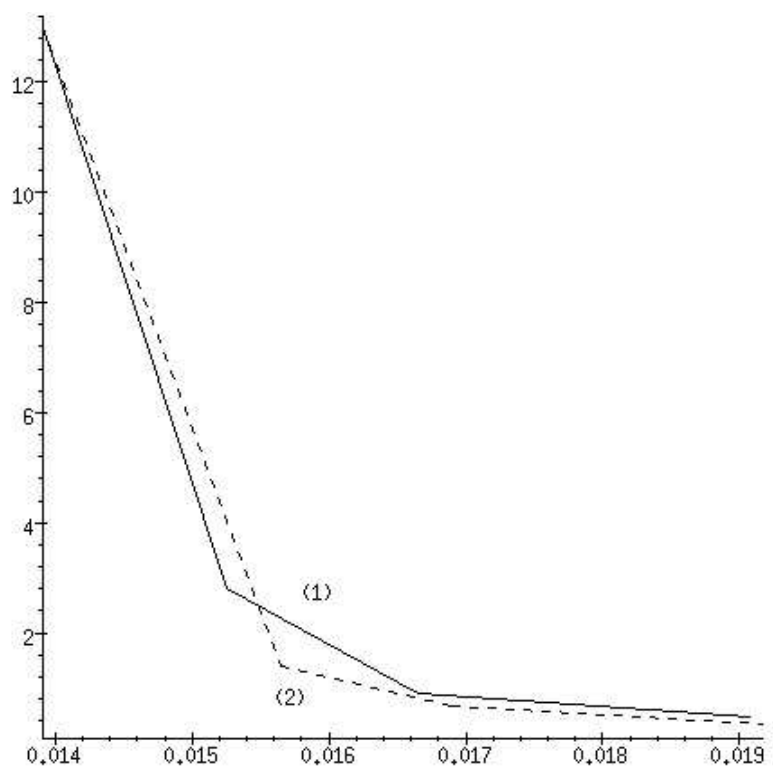

Figure 12: Norm of the control points box deformation versus least-squares approximation error between the models and the data. The 2 curves correspond to a regularization with SVD or with a regularizing term. 


\section{Application to the representation of 3D medical data}

We present in this section applications of the algorithm on 3D cardiac images. Those images have been obtained with nuclear medicine (SPECT images) and volumetric scanner X (Dynamic Spatial Reconstructor - Mayo Clinic).

The SPECT images (Single Photon Emission Computed Tomography) are obtained by the measure of the electromagnetic radiation coming from radioactive isotopes injected in the human body. This kind of images permits to get informations about the metabolic behavior of organs. There are functional images. The SPECT MIBI image (with corresponds to a new myocardial perfusion tracer, technium $99 \mathrm{~m} \mathrm{MIBI)}$ is a volume of $64 \times 64 \times 64$ voxels. The SPECT image is a volume of size $64 \times 64 \times 21$.

The DSR image has a very good resolution $(98 \times 100 \times 110$ for a voxel of $0.926 \mathrm{~mm} 3)$. It was obtained by injection of a contrast product in the ventricle, which allows to distinguish clearly the cavity.

The $3 \mathrm{D}$ original data are visualized as a series of $2 \mathrm{D}$ cross-sections (according to the $\mathrm{Z}$ axis) on Figures 13 and 14. 


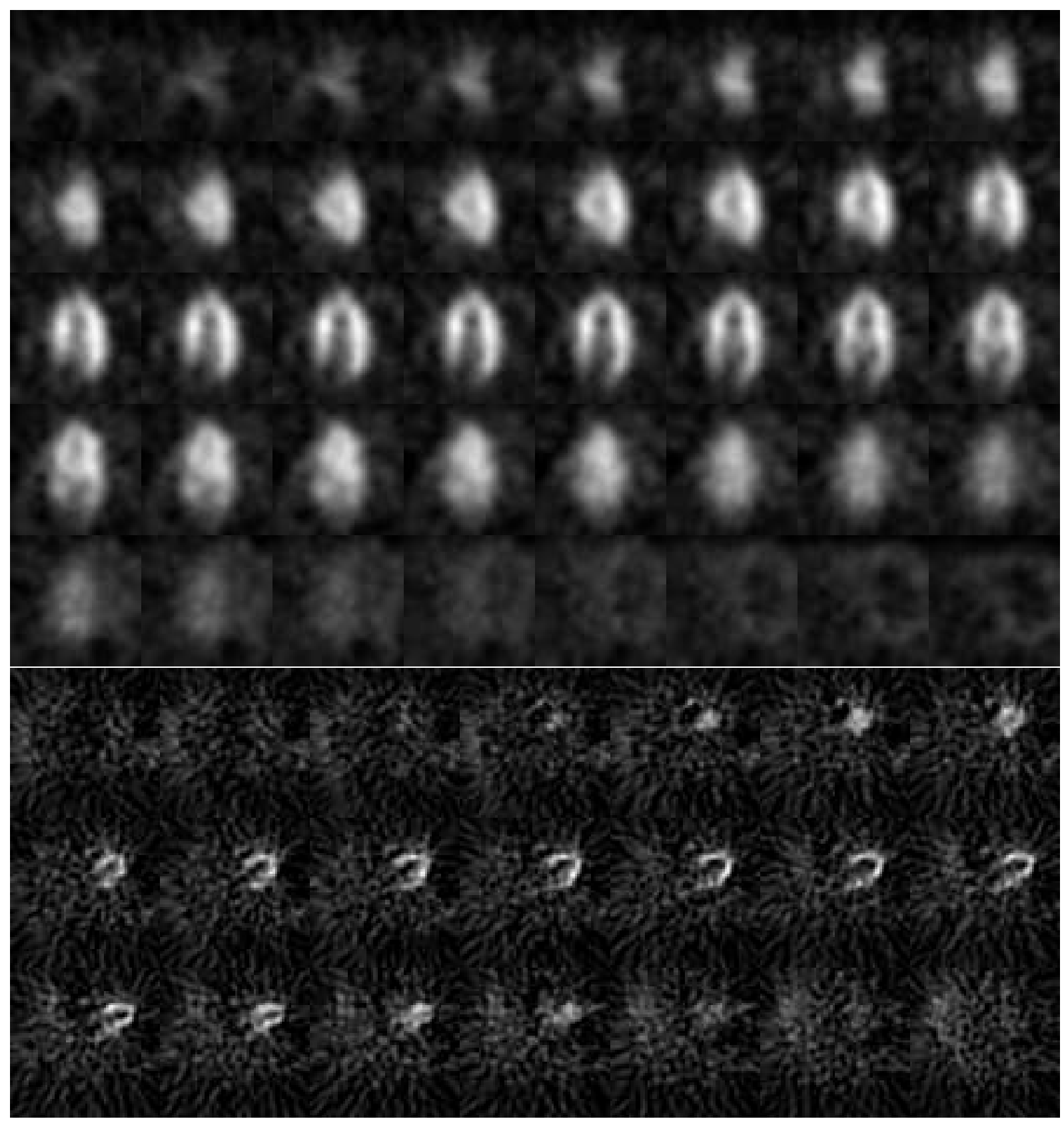

Figure 13: 3D images of the left ventricle (order of cross-sections: from left to right and from top to bottom). Top: SPECT MIBI - SOPHA Medical. Bottom: SPECT - FOCUS Medical. 


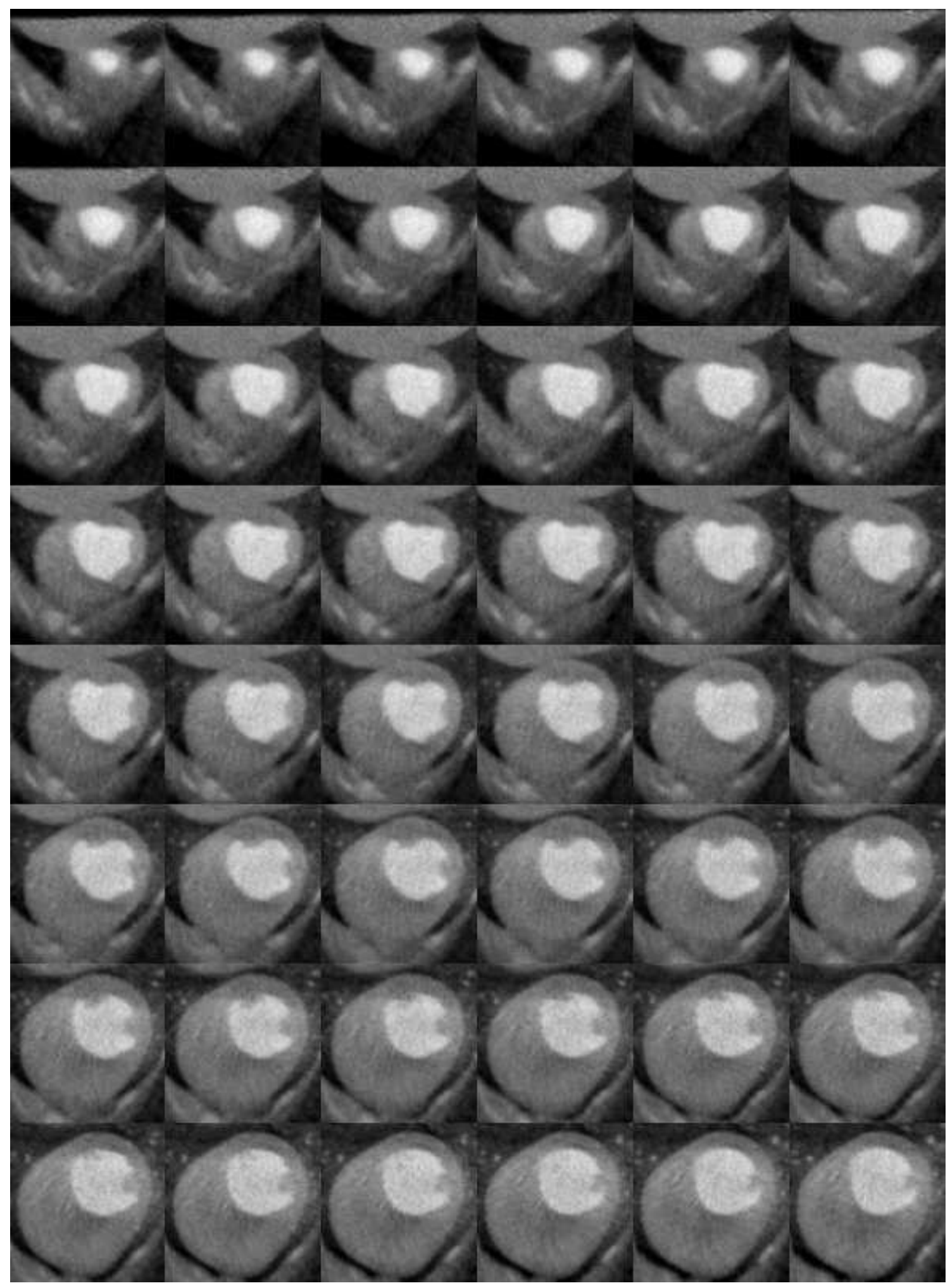

Figure 14: 3D image of the left ventricle (order of cross-sections: from left to right and from top to bottom). (Scanner X - DSR). 


\section{Morphological segmentation of the data}

In order to get a set of $3 \mathrm{D}$ points which corresponds to the anatomical structure that we want to study (epicardium or endocardium or midwall of the cardiac left ventricle), and therefore fit our model on this set of points, we have to segment the original 3D data. As one can visually remark on Figures 13 and 14, this is not an easy task. The SPECT images are very noisy, and, on the DSR image, the segmentation of the external wall of the ventricle (epicardium) cannot be done with a simple thresholding.

To obtain an accurate and robust segmentation, we must combine thresholding with mathematical morphology and connected components analysis (as in Hoehne [36]). We first choose a threshold which grossly separates the ventricle (high values in SPECT and DSR images) from the rest of the image. Then we choose the largest connected component in the resulting binary image, and perform a equal number of erosions and dilations (morphological closings). This last operation is necessary to bridge little gaps (in particular for the epicardium on the upper part of the DSR image) and smooth the overall segmentation. Finally, the extraction of an isosurface from that last image provides the set of $3 \mathrm{D}$ points that we need as input for the complete reconstruction algorithm.

Results of those operations are presented on Figures 15, 16 and 17. For a correct estimation of the quality of those segmentations, we superimposed the segmented surface on the image (we present cross-sections along the $\mathrm{Z}$ axis).

\section{Fit of the model}

We applied on those data points sets a parametric model defined by a box of control points of size $5 \times 5 \times 5$, and the 11 parameters which describe the initial superellipsoid. We used the iterative two-step algorithm detailed above (see section (3.3)), with 30 iterations. The results are presented on Figures 15, 16 and 18 (cross-sections along the $\mathrm{Z}$ axis).

\section{Visualization of the data and model surfaces}

The corresponding surfaces of the previous images are shown in this section. Figure 19 shows three different views of the SPECT MIBI data and of the figure 15. Figure 20 corresponds to the SPECT data and to Figure 16. Figure 21 (two different views) corresponds to the DSR data and to Figure 18. 


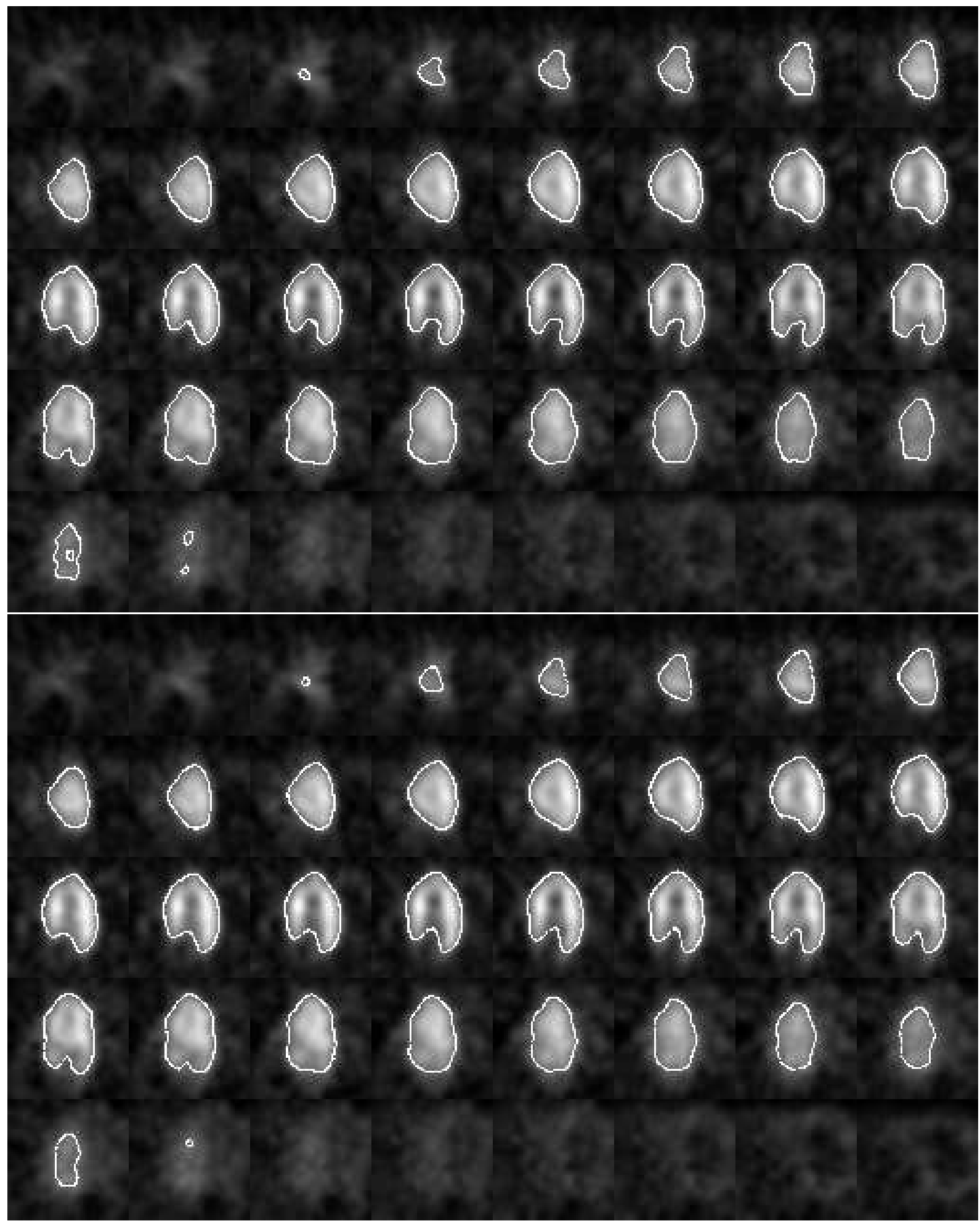

Figure 15: Segmentation and representation of the epicardium (external wall of the ventricle) for the 3D image of the left ventricle (SPECT MIBI). Top: isosurface superimposed to the image. Bottom: reconstruction by the model. 


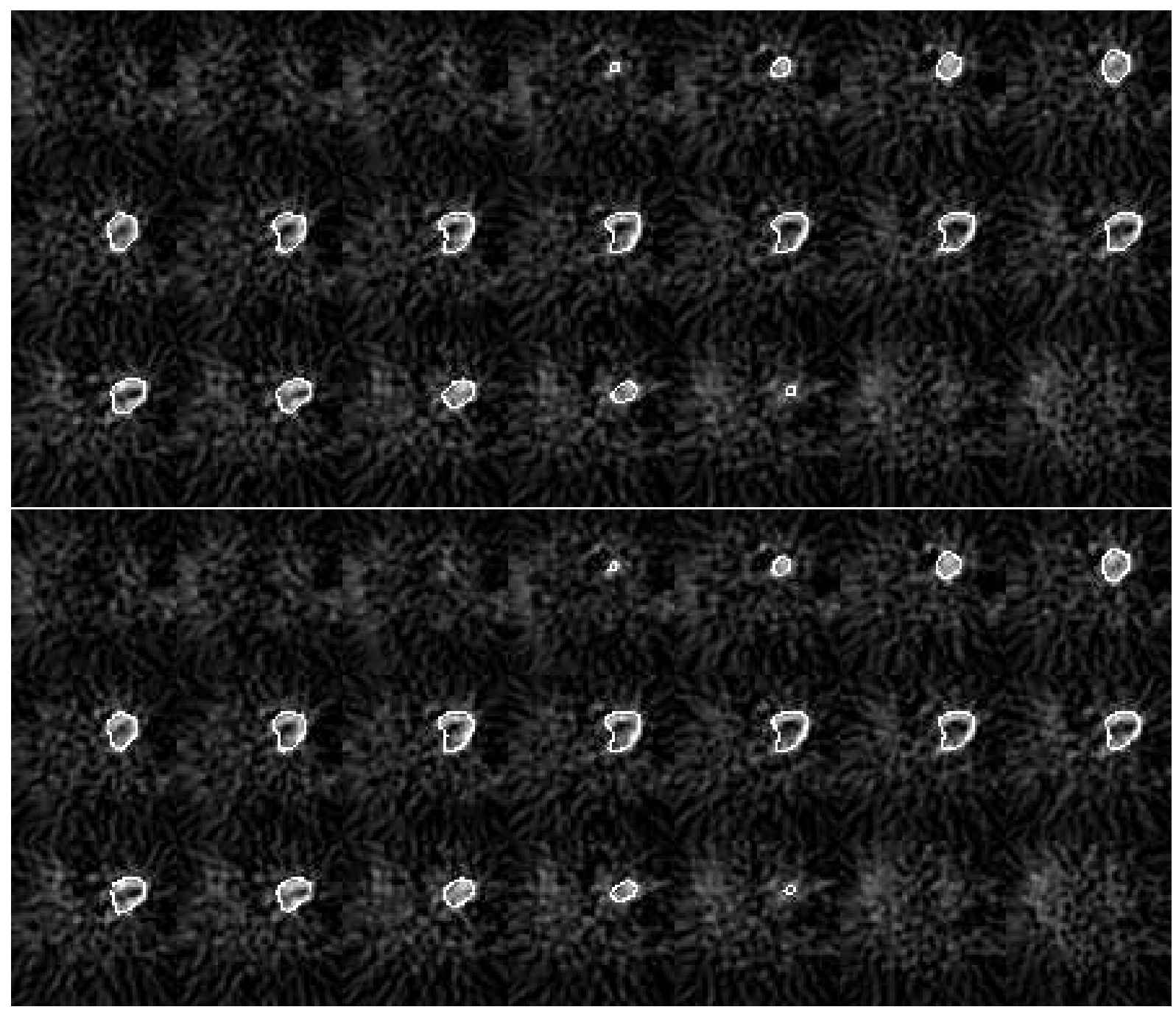

Figure 16: Segmentation and representation of the epicardium (external wall of the ventricle)for the 3D image of the left ventricle (SPECT - FOCUS Medical). Top: isosurface superimposed to the image. Bottom: reconstruction by the model. 


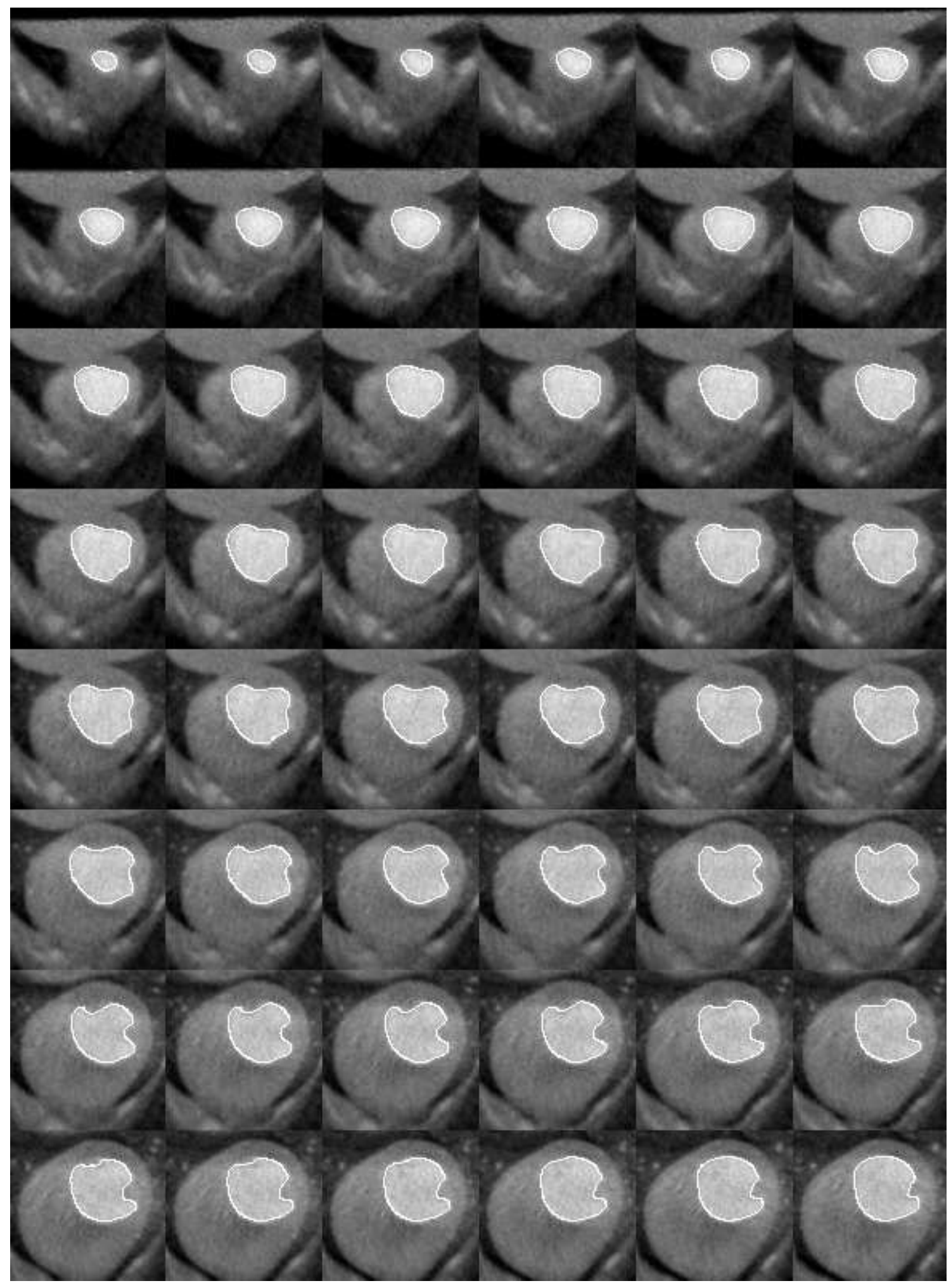

Figure 17: Segmentation of the endocardium (internal wall of the ventricle) for the 3D image of the left ventricle (Scanner X - DSR). 


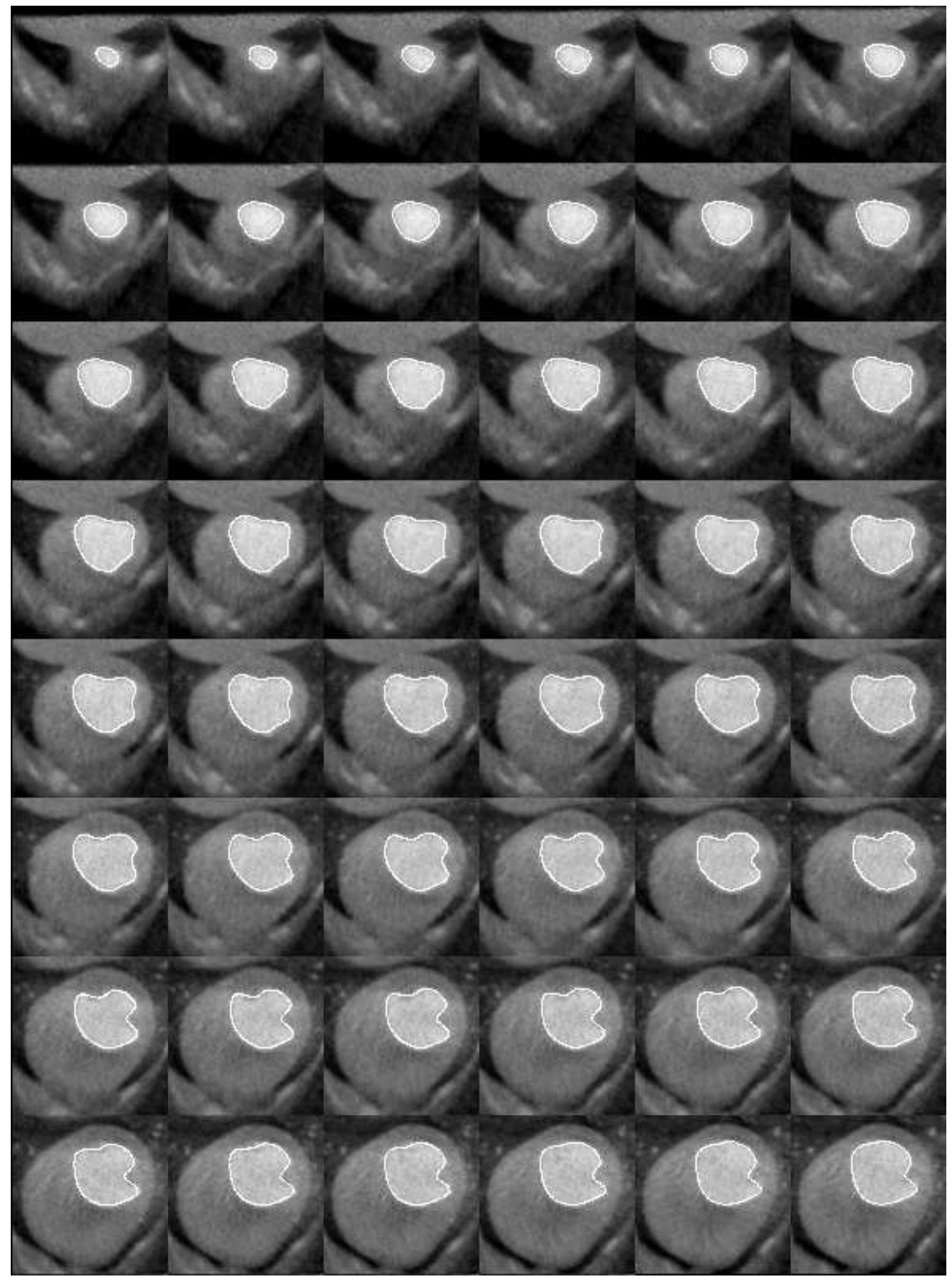

Figure 18: Reconstruction of the endocardium by the model. 

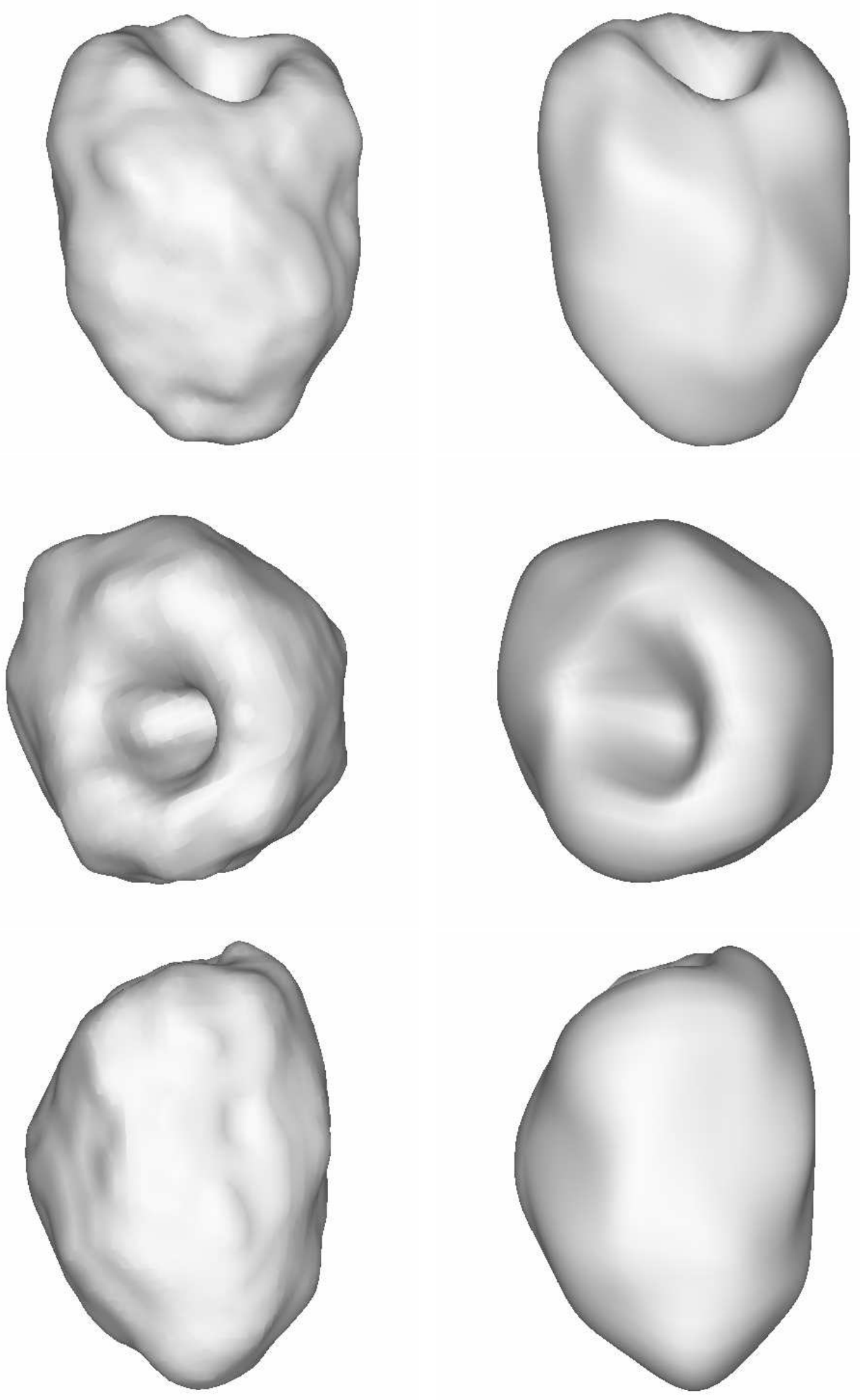

Figure 19: Left: isosurface from the segmentation of SPECT MIBI data (6000 points). Right: representation by the parametric model (defined by 130 points). Compression ratio: 46. (3 view points) 

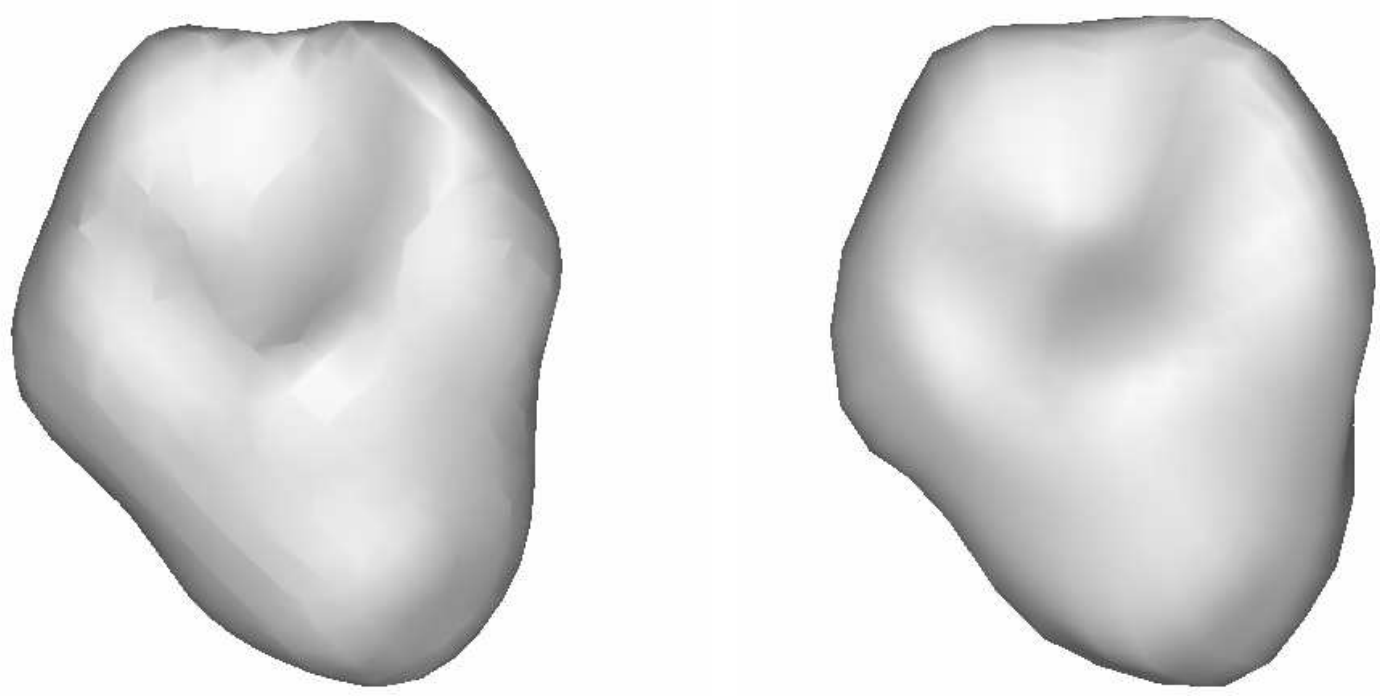

Figure 20: Left: isosurface from the segmentation of SPECT data (1000 points). Right: representation by the parametric model (defined by 130 points). Compression ratio: 7.7.

\section{Compression}

The isosurfaces obtained by morphological segmentation are composed respectively of 6000 (SPECT MIBI, Figure 19), 1000 (SPECT, Figure 20) and 20.000 (DSR, Figure 21) 3D points.

With the representation of those data by a parametric model defined by $1303 \mathrm{D}$ points, we reduce the information which is needed for the description of the ventricles by a ratio of $46,7.7$ and 77 respectively for SPECT MIBI, SPECT and DSR data. Note that for the simultaneous computation of one model for the epicardium and endocardium (DSR), we get a compression ratio of 154 .

The two diagrams of figure 22 represent the evolution of the least-squares error $\|\boldsymbol{B P}-\boldsymbol{X}\|$ versus the number of iterations as well as the evolution of the deformation of the box of control points $\left\|\boldsymbol{P}_{n+1}-\boldsymbol{P}_{n}\right\|$ (each curve represents a different volume of data). 

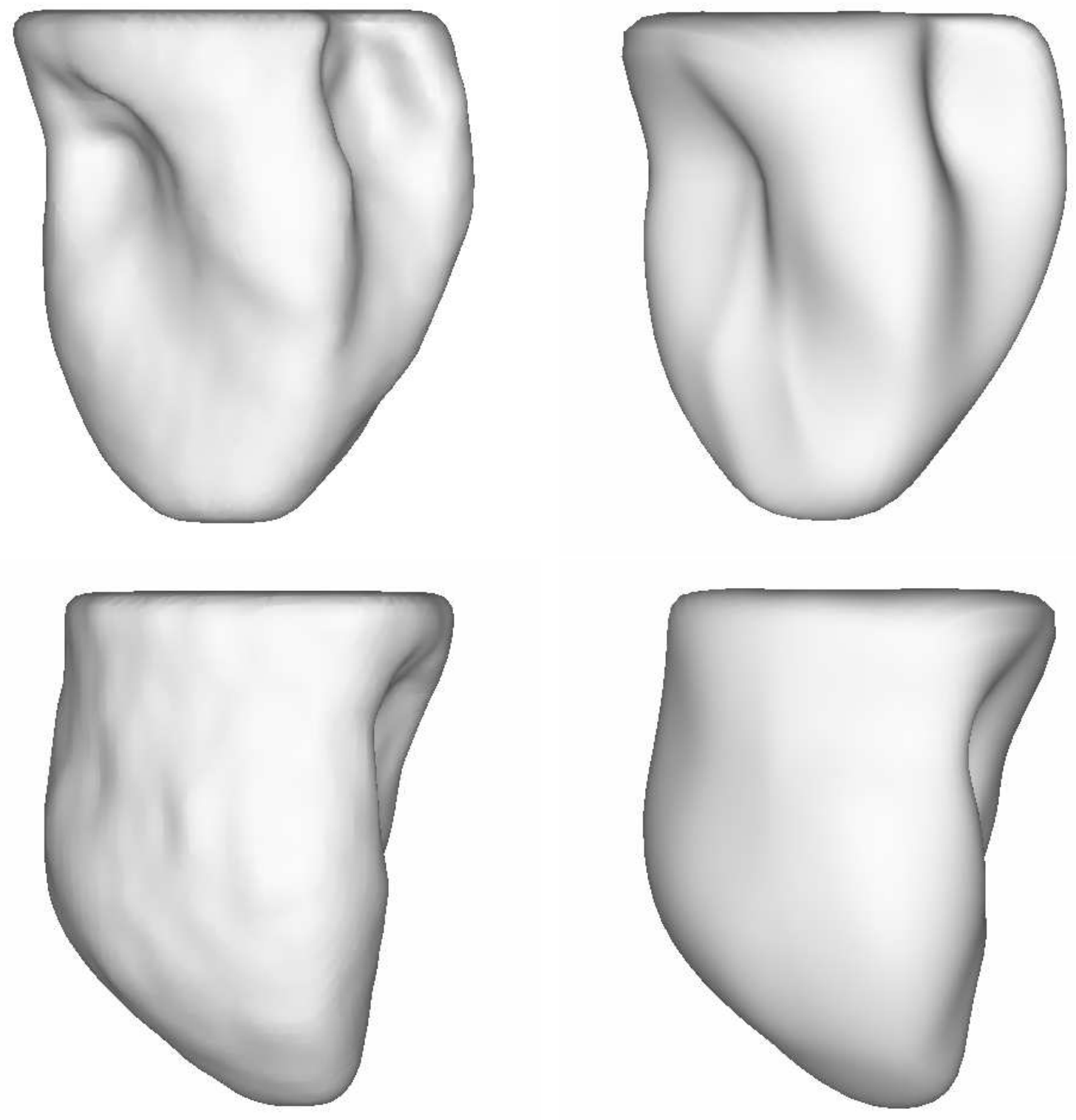

Figure 21: Left: isosurface (endocardium) from the segmentation of DSR data (10.000 points for the surface). Right: representation by a parametric model, defined by 130 points. Compression ratio: 77. (2 view points) 

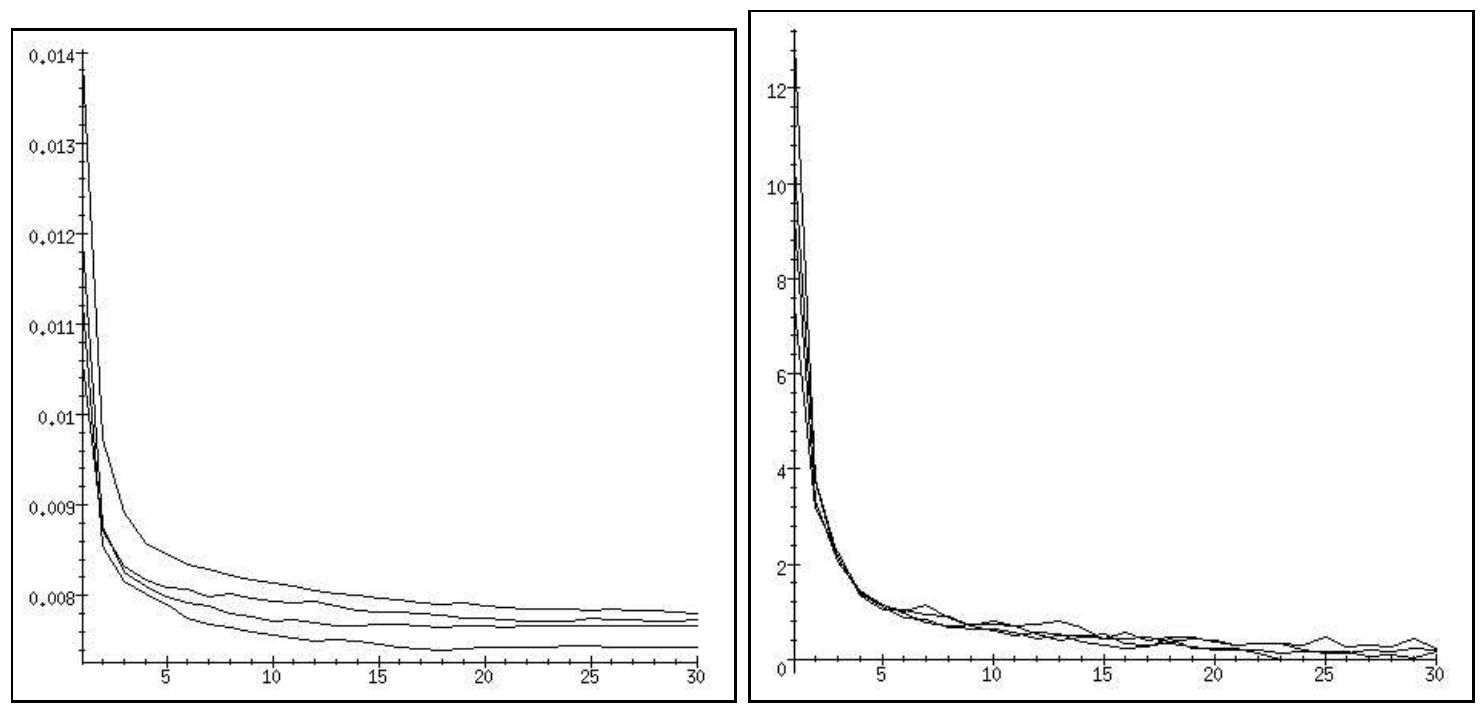

Figure 22: Left: Evolution of the least-squares approximation error between data and the models versus the number of iterations (the 4 curves correspond to 4 different models). Right: Evolution of the deformation of the box of control points versus the number of iterations (the 4 curves correspond to 4 different models).

\section{Conclusion}

We presented a new approach to shape reconstruction applied to unstructured 3D data. It is based on a first approximation giving the best fit with a superquadric model. This is followed by a two-step algorithm for refining the details of the previous shape by making use of free-form deformations. The data are a set of points in a 3-D image, and the closest point to data is used for the definition of a displacement field. We also presented two methods to control the regularity of the control points box defining the free-form deformations, using singular value decomposition or adding a regularizing term in the previous algorithm. The main advantage of this approach is the compactness of the description of a complex shape, and also the robustness of the fit even in the presence of outliers or sparse data.

Experimental results have been shown for a various number of 3D cardiac images to capture the 3D shape of the left ventricle.

Future work will study the use of this compact and robust model for the dynamic analysis of 4D temporal series of 3D images of the beating heart. This will be presented in a companion paper [9]. 


\section{Acknowledgments}

We would like to thank Serge Benayoun and Alexis Gourdon who provided us with substantial help through fruitful discussions and Grégoire Malandain for his judicious remarks about segmentation with morphological operators. Thanks to Dr. R. Robb and D. Hanson, Biomedical Imaging Resource, Mayo Foundation/Clinic, to Sopha Medical and to Focus Medical for the data. This work was partially supported by Digital Equipment Corporation.

\section{A Additional details about superquadrics}

\section{A.1 Definition}

Superquadrics are obtained by the spherical product (see [11]) of two 2D curves. The superellipsoid is the spherical product of the superellipse with itself. The curve defined by the implicit equation:

$$
\left(\frac{x}{a}\right)^{\frac{2}{\epsilon}}+\left(\frac{y}{b}\right)^{\frac{2}{\epsilon}}=1
$$

is a superellipse. A natural parameterization is:

$$
\left\{\begin{array}{l}
x=a \cos ^{\epsilon} \theta, \quad-\pi \leq \theta<\pi \\
y=b \sin ^{\epsilon} \theta
\end{array}\right.
$$

For simplification in the formulation in the above equation and the followings, the powers of the $\cos , \sin$ and $\tan$ functions are to take in the following sense:

$$
u^{\epsilon}=\operatorname{sign}(u)|u|^{\epsilon}=u|u|^{\epsilon-1}
$$

In the implicit equation, powers $\frac{2}{\epsilon}$ means:

$$
u^{\frac{2}{\epsilon}}=\left(u^{2}\right)^{\frac{1}{\epsilon}}=|u|^{\frac{2}{\epsilon}} .
$$

The $\epsilon$ coefficient controls the squareness of the curves: courbes :

- $\epsilon$ small, the shape is close to a square;

- $\epsilon=1$, it is a circle;

- $\epsilon=2$ the shape has flat bevel;

- $\epsilon$ large, the shape is pinched.

The spherical product of these curves gives a uniform mathematical formulation of the family of superquadrics. 
Remark : Parameters $\eta$ and $\omega$ correspond to the latitude and longitude of vector $\boldsymbol{x}$ in spherical components; parameters $a_{1}, a_{2}$ and $a_{3}$ define the size of the superellipsoid along $x, y$ and $z$ axes; $\epsilon_{1}$ and $\epsilon_{2}$ define the shape of the superquadric along latitude and longitude. Some exemples are presented figure 1.

\section{A.2 Rigid transform}

All the equations of the previous section are defined in a intrinsic local frame with the center of the superellipsoid as origin. To fit the superellipsoid on the data, we must be able to position it anywhere in space, that means rewrite those equations in a general frame $R_{O}$.

A homogeneous rigid transform $T$ is defined by its matrix:

$$
T=\left[\begin{array}{cc}
R & t \\
0 & 1
\end{array}\right],
$$

where $R$ is a $3 \times 3$ rotation matrix and $\boldsymbol{t}$ a $3 \times 1$ translation vector. We note $\boldsymbol{x}$ and $\underline{\boldsymbol{x}}$ the position vectors respectively in the object frame and in frame $R_{O}$. We have then:

$$
\begin{aligned}
& \underline{\boldsymbol{x}}=R \boldsymbol{x}+\boldsymbol{t}, \\
& \boldsymbol{x}=R^{t}(\underline{\boldsymbol{x}}-\boldsymbol{t}),
\end{aligned}
$$

since the inverse $R^{-1}$ is equal to $R^{t}$.

We choose the 3 Euler angles $\varphi, \theta$ and $\psi$ to represent a rotation in $\mathbb{R}^{3}$. The new inside-outside function is obtained by replacing $\boldsymbol{x}$ by $T^{-1}(\underline{\boldsymbol{x}})$ in 1 :

$$
\widehat{F}(\underline{\boldsymbol{x}})=F\left(T^{-1}(\underline{\boldsymbol{x}})\right) \text {. }
$$

Function $\widehat{F}$ now depends on 11 parameters: $\left(a_{1}, a_{2}, a_{3}, \epsilon_{1}, \epsilon_{2}, \varphi, \theta, \psi, t_{x}, t_{y}, t_{z}\right)$. We use this function to fit the data with the superellipsoid.

\section{A.3 Initialization of the fit}

It is a two-step algorithm. The first step consists in the initialization of the parametric surface from the 3D data points set:

The initial superquadric is an ellipsoid (parameters $\epsilon_{1}$ and $\epsilon_{2}$ equal to 1 ). It is centered at the center of gravity of the data set. Its orientation is defined by the moments of inertia of the data, computed from the matrix of second order centered moments $M$ :

$$
M=\frac{1}{N} \sum_{i=1}^{N}\left[\begin{array}{ccc}
\left(y_{i}-\bar{y}\right)^{2}+\left(z_{i}-\bar{z}\right)^{2} & -\left(y_{i}-\bar{y}\right)\left(x_{i}-\bar{x}\right) & -\left(z_{i}-\bar{z}\right)\left(x_{i}-\bar{x}\right) \\
-\left(x_{i}-\bar{x}\right)\left(y_{i}-\bar{y}\right) & \left(x_{i}-\bar{x}\right)^{2}+\left(z_{i}-\bar{z}\right)^{2} & -\left(z_{i}-\bar{z}\right)\left(y_{i}-\bar{y}\right) \\
-\left(x_{i}-\bar{x}\right)\left(z_{i}-\bar{z}\right) & -\left(y_{i}-\bar{y}\right)\left(z_{i}-\bar{z}\right) & \left(x_{i}-\bar{x}\right)^{2}+\left(y_{i}-\bar{y}\right)^{2}
\end{array}\right] .
$$


The center of gravity and the 3 axes of inertia define the new frame of the object. To compute the size of the three axes, we compare the matrix of an ellipsoid surface $J$ and $M$ after diagonalization. The form of $J$ is:

$$
J=\frac{\mu}{3}\left[\begin{array}{ccc}
a_{2}^{2}+a_{3}^{2} & 0 & 0 \\
0 & a_{3}^{2}+a_{1}^{2} & 0 \\
0 & 0 & a_{1}^{2}+a_{2}^{2}
\end{array}\right],
$$

where $a, b$ and $c$ are the sizes of the axes and $\mu$ is the mass (here 1). Hence, if $\lambda_{1}, \lambda_{2}$ and $\lambda_{3}$ are the eigen values of $M$, the sizes of the axes are computed as follows:

$$
\left\{\begin{array}{l}
a_{1}^{2}=\frac{3}{2 \mu}\left(\lambda_{2}+\lambda_{3}-\lambda_{1}\right), \\
a_{2}^{2}=\frac{3}{2 \mu}\left(\lambda_{1}+\lambda_{3}-\lambda_{2}\right), \\
a_{3}^{2}=\frac{3}{2 \mu}\left(\lambda_{1}+\lambda_{2}-\lambda_{3}\right) .
\end{array}\right.
$$

\section{B Definition of FFDs}

The FFD formulation is divided into two steps:

1. Computation of the local coordinates of the object points in the frame defined by the box of control points. We define a coordinate system in the local frame associated to the parallelepipedic box in which the object to be deformed is embedded. The origin of the frame is a vertex $\boldsymbol{X}_{\mathbf{0}}$ of the box, and the axes of this frame are the 3 edges $(\boldsymbol{S}, \boldsymbol{T}, \boldsymbol{U})$ of the box coming from $\boldsymbol{X}_{\mathbf{0}}$. Every $3 \mathrm{D}$ point $\boldsymbol{X}$ has $(s, t, u)$ coordinates in this system such as:

$$
\boldsymbol{X}=\boldsymbol{X}_{\mathbf{0}}+s \boldsymbol{S}+t \boldsymbol{T}+u \boldsymbol{U},
$$

where $s, t, u$ are given by:

$$
s=\frac{\boldsymbol{S} \cdot\left(\boldsymbol{X}-\boldsymbol{X}_{\mathbf{0}}\right)}{\boldsymbol{S} \cdot \boldsymbol{S}}, t=\frac{\boldsymbol{T} \cdot\left(\boldsymbol{X}-\boldsymbol{X}_{\mathbf{0}}\right)}{\boldsymbol{T} \cdot \boldsymbol{T}}, u=\frac{\boldsymbol{U} \cdot\left(\boldsymbol{X}-\boldsymbol{X}_{\mathbf{0}}\right)}{\boldsymbol{U} \cdot \boldsymbol{U}},
$$

Remark that $\boldsymbol{X}$ is inside the box iff $s, t$ and $u$ are in $[0,1]$.

We then generate a grid of $(l+1)(m+1)(n+1)$ control points in the box by setting :

$$
\boldsymbol{P}_{\boldsymbol{i j k}}=\boldsymbol{X}_{\mathbf{0}}+\frac{i}{l} \boldsymbol{S}+\frac{j}{m} \boldsymbol{T}+\frac{k}{n} \boldsymbol{U} .
$$

The object is linked to the grid of control points by a deformation function defined as a tensor product of trivariate Bernstein polynomials (polynomials associated to Bezier curves). The position $\boldsymbol{X}$ of an arbitrary point is evaluated by the computation of its local coordinates $(s, t, u)$, and of the trivariate vector polynomial:

$$
\boldsymbol{X}=\sum_{i=0}^{l} \sum_{j=0}^{m} \sum_{k=0}^{n} C_{l}^{i} C_{m}^{j} C_{n}^{k}(1-s)^{l-i} s^{i}(1-t)^{m-j} t^{j}(1-u)^{n-k} u^{k} \boldsymbol{P}_{\boldsymbol{i j k}}
$$


2. Displacement of the control points and estimation of the new position of the deformed object. The deformation of the object is specified by moving the control points $\boldsymbol{P}_{\boldsymbol{i} \boldsymbol{j} \boldsymbol{k}}$ from their latticial position to a new position $\boldsymbol{P}_{\boldsymbol{i j} \boldsymbol{k}}^{\boldsymbol{*}}$. The position of a point $\boldsymbol{X}$ of the deformed object is then computed with the previous vector polynomial:

$$
\boldsymbol{X}^{*}=\sum_{i=0}^{l} \sum_{j=0}^{m} \sum_{k=0}^{n} C_{l}^{i} C_{m}^{j} C_{n}^{k}:(1-s)^{l-i} s^{i}(1-t)^{m-j} t^{j}(1-u)^{n-k} u^{k} \boldsymbol{P}_{\boldsymbol{i j k}}^{*}
$$

\section{References}

[1] A. Amini and J. Duncan. Bending and stretching models for LV wall motion analysis from curves and surfaces. Image and vision computing, 10:418-430, August 1992.

[2] A. Amini, R. Owen, P. Anandan, and J. Duncan. Non-rigid motion models for tracking the left ventricular wall. In Information processing in medical images, Lecture notes in computer science, pages 343-357, 1991. Springer-Verlag.

[3] N. Ayache. Medical computer vision, virtual reality and robotics. Image and Vision Computing, 13(4):295-313, May 1995.

[4] N. Ayache, J.D. Boissonnat, E. Brunet, L. Cohen, J.P. Chièze, B. Geiger, O. Monga, J.M. Rocchisani, and P. Sander. Building highly structured volume representations in 3D medical images. In Computer Aided Radiology, June 1989. Berlin, West-Germany.

[5] N. Ayache, J.D. Boissonnat, L. Cohen, B. Geiger, O. Monga, J. Levy-Vehel, and P. Sander. Steps toward the automatic interpretation of 3D images. NATO ASI Series on $3 D$ Imaging in Medicine, $\mathrm{F}$ 60:107-120, 1990.

[6] N. Ayache, I. Cohen, and I. Herlin. Medical Image Tracking, chapter 20. MIT Press, 1992.

[7] R. Bajcsy and F. Solina. Three dimensional object representation revisited. In Proceedings IEEE International Conference on Computer Vision (ICCV), pages 231-240, London, June 1987.

[8] E. Bardinet, L.D. Cohen, and N. Ayache. A parametric deformable model to fit unstructured 3D data. Research report 2617, INRIA, Sophia-Antipolis, July 1995.

[9] E. Bardinet, L.D. Cohen, and N. Ayache. Tracking and motion analysis of the left ventricle with deforble superquadrics. Medical Image Analysis, 1(2), 1996. Also INRIA Research Report RR-2797, INRIA Sophia Antipolis, February 1996.

[10] E. Bardinet, L.D. Cohen, and N. Ayache. Tracking medical 3D data with a deformable parametric model. In Proceedings European Conference on Computer Vision (ECCV), April 1996.

[11] A. Barr. Superquadrics and angle-preserving deformations. IEEE Computer Graphics and Applications, 1(1):11-23, January 1981. 
[12] A. Barr. Global and local deformations of solid primitives. Computer Graphics (Proceedings SIGGRAPH'84), 18(3):21-30, July 1984.

[13] S. Benayoun, C. Nastar, and N. Ayache. Dense non-rigid motion estimation in sequences of 3D images using differential constraints. In Proceedings Conference on Computer Vision, Virtual Reality and Robotics in Medecine (CVRMed), pages 309-318, Nice, France, April 1995.

[14] A. Blake and A. Zisserman. Visual Reconstruction. MIT Press, 1987.

[15] G. Borgefors. Distance transformations in arbitrary dimensions. Computer Vision, Graphics, and Image Processing, 27:321-345, 1984.

[16] T. Boult and A. Gross. Recovery of superquadrics from depth information. In Proceedings Workshop on Spatial Reasoning and Multi-Sensor Fusion, pages 128-137, Saint Charles, Illinois, October 1987.

[17] Y.K. Chang and A. Rockwood. A generalized de casteljau approach to 3D free-form deformation. Computer Graphics (Proceedings SIGGRAPH'94), pages 257-260, July 1994.

[18] C. Chen, J. Luo, K. Parker, and T.S. Huang. CT volumetric data-based left ventricle motion estimation: an integrated approach. International Journal of Computer Vision, 11:85-100, October 1993.

[19] I. Cohen and L.D. Cohen. A hybrid hyperquadric model for 2-D and 3-D data fitting. In Proceedings International Conference on Pattern Recognition (ICPR), pages B-403-405, Jerusalem, October 1994. IEEE. Part of Inria TR 2188. See also in Computer Vision and Image Understanding (63) 3:527-541, May 1996.

[20] I. Cohen, L.D. Cohen, and N. Ayache. Using deformable surfaces to segment 3-D images and infer differential structures. Computer Vision, Graphics, and Image Processing: Image Understanding, 56(2):242-263, September 1992.

[21] L.D. Cohen. On active contour models and balloons. Computer Vision, Graphics, and Image Processing: Image Understanding, 53(2):211-218, March 1991. INRIA TR 1075, August 1989.

[22] L.D. Cohen. Auxiliary variables and two-step iterative algorithms in computer vision problems. Journal of Mathematical Imaging and Vision, 6(1):61-86, January 1996. See also ICCV'95.

[23] L.D. Cohen and I. Cohen. Finite element methods for active contour models and balloons for 2-D and 3-D images. IEEE Transactions on Pattern Analysis and Machine Intelligence, 15(11), November 1993. Cahiers de Mathématiques de la Décision 9124 (CEREMADE).

[24] S. Coquillart. Extended free-form deformation: A sculpturing tool for 3D geometric modeling. Computer Graphics (Proceedings SIGGRAPH'90), 24(4):187-196, August 1990.

[25] S. Coquillart and P. Jancene. Animated free-form deformation: an interactive animation technique. Computer Graphics (Proceedings SIGGRAPH'91), 25(4):23-26, July 1991.

[26] P.E. Danielsson. Euclidean distance mapping. Computer Graphics And Image Processing, 14:227-248, 1980. 
[27] H. Delingette, M. Hebert, and K. Ikeuchi. Shape representation and image segmentation using deformable surfaces. In Proceedings IEEE Computer Society Computer Vision and Pattern Recognition (CVPR), Maui, Hawai, June 1991.

[28] F. Ferrie, J. Lagarde, and P. Whaite. Darboux frames, snakes and superquadrics: geometry from the bottom-up. In Proceedings IEEE Workshop on Interpretation of $3 D$ Scenes, pages 170-176, Austin, November 1989.

[29] F. Ferrie, J. Lagarde, and P. Whaite. Recovery of volumetric object descriptions from laser rangefinder images. In Proceedings European Conference on Computer Vision (ECCV), pages 387-396, Antibes, France, April 1990.

[30] M. Gardner. The superellipse: a curve that lies between the ellipse and the rectangle. Scientific American, 213:222-234, September 1965.

[31] J. Griessmair and W. Purgathofer. Deformation of solids with trivariate b-splines. In North-Holland Elsevier Science Publishers, editor, Eurographics'89, pages 187-193, 1989.

[32] W. E. L. Grimson. From Images to Surfaces: A computational study of the Human Early vision system. The MIT Press, 1981.

[33] A. Gupta and R. Bajcsy. An integrated approach for surface and volumetric segmentation of range images using biquadrics and superquadrics. SPIE Applications of artificial intelligence X: Machine Vision and Robotics, 1708:210-227, 1992.

[34] A. Gupta and R. Bajcsy. Volumetric segmentation of range images of 3D objects using superquadric models. Computer Vision, Graphics, and Image Processing: Image Understanding, 58(3):302-326, November 1993.

[35] S. Han, D. Goldgof, and K. Bowyer. Using hyperquadrics for shape recovery from range data. In Proceedings IEEE International Conference on Computer Vision (ICCV), pages 492-496, Berlin, June 1993.

[36] K. Höhne and W. Hanson. Interactive 3D segmentation of MRI and CT volumes using morphological operations. Journal of Computer Assisted Tomography, 16(2):285-294, March 1992.

[37] W. Hsu, J. Hughes, and H. Kaufman. Direct manipulation of free-form deformations. Computer Graphics (Proceedings SIGGRAPH'92), 26(2):177-184, July 1992.

[38] M. Kass, A. Witkin, and D. Terzopoulos. Snakes: active contour models. International Journal of Computer Vision, 1:321-331, 1987.

[39] H. Lamousin and W. Waggenspack. Nurbs-based free-form deformations. IEEE Computer Graphics and Applications, 14(6):59-65, November 1994.

[40] F. Leitner and P. Cinquin. Dynamic segmentation: Detecting complex topology 3D-object. In Proceedings International Conference of the IEEE Engineering in Medicine and Biology Society, pages 295-296, Orlando, Florida, November 1991. 
[41] D. Metaxas and S. Dickinson. Integration of quantitative and qualitative techniques for deformable model fitting from orthographic, perspective and stereo projections. In Proceedings IEEE International Conference on Computer Vision (ICCV), pages 641-649, Berlin, Germany, May 1993.

[42] D. Metaxas and D. Terzopoulos. Shape and nonrigid motion estimation through physics-based synthesis. IEEE Transactions on Pattern Analysis and Machine Intelligence, pages 580-591, June 1993.

[43] S.K. Mishra, D.M. Goldgof, and T.S. Huang. Motion analysis and epicardial deformation estimation from angiography data. In Proceedings IEEE Computer Society Computer Vision and Pattern Recognition (CVPR), pages 331-336, Maui, Hawaii, June 1991.

[44] C. Nastar and N. Ayache. Spatio-temporal analysis of nonrigid motion from 4D data. In Proceedings IEEE Workshop on non-rigid and articulated objects, pages 146-151, Austin, November 1994.

[45] J. Park, D. Metaxas, and A. Young. Deformable models with parameter functions : application to heart-wall modeling. In Proceedings IEEE Computer Society Computer Vision and Pattern Recognition (CVPR), pages 437-442, June 1994.

[46] S. Parry. Free-form deformations in a constructive solid geometry modeling system. PhD thesis, Department of Civil Engineering, Brigham Young University, April 1986.

[47] A. Pentland. Perceptual organization and the representation of natural form. Artificial Intelligence, 28:293-331, 1986.

[48] A. Pentland. Extraction of deformable part models. In Proceedings European Conference on Computer Vision (ECCV), pages 397-401, Antibes, France, April 1990.

[49] A. Pentland and S. Sclaroff. Closed-form solutions for physically-based shape modeling and recognition. IEEE Transactions on Pattern Analysis and Machine Intelligence, 13(7):715-729, July 1991.

[50] W.H. Press, B.P. Flannery, S.A. Teukolsky, and W.T. Vetterling. Numerical recipies in C, the art of scientific computing. Cambridge University Press, 1989.

[51] T.W. Sederberg and S.R. Parry. Free-form deformation of solid geometric models. Computer Graphics (Proceedings SIGGRAPH'86), 20(4):151-160, August 1986.

[52] P. Shi, A. Amini, G. Robinson, A. Sinusas, C. Constable, and J. Duncan. Shape-based 4D left ventricular myocardial function analysis. In Proceedings IEEE Workshop on Biomedical Image Analysis (WBIA), pages 88-97, Seattle, June 1994.

[53] F. Solina and R. Bajcsy. Range image interpretation of mail pieces with superquadrics. In $A A A I$, pages 733-737, Seattle, 1987.

[54] F. Solina and R. Bajcsy. Recovery of parametric models from range images: The case for superquadrics with global deformations. IEEE Transactions on Pattern Analysis and Machine Intelligence, 12(2):131147, February 1990. 
[55] R. Szeliski and S. Lavallée. Matching 3D anatomical surfaces with non-rigid deformations using octreesplines. In Proceedings SPIE Geometric Methods in Computer Vision II, volume 2031, pages 306-315, San Diego, July 1993. Society of Photo-Optical Instrumentation Engineers.

[56] R. Szeliski and S. Lavallée. Matching 3-d anatomical surfaces with non-rigid deformations using octreesplines. In Proceedings IEEE Workshop on Biomedical Image Analysis (WBIA), Seattle, Washington, June 1994.

[57] G. Taubin. An improved algorithm for algebraic curve and surface fitting. In Proceedings IEEE International Conference on Computer Vision (ICCV), pages 658-665, Berlin, 1993.

[58] D. Terzopoulos. The computation of visible-surface representations. IEEE Transactions on Pattern Analysis and Machine Intelligence, 10(4):417-438, July 1988.

[59] D. Terzopoulos and D. Metaxas. Dynamic 3D models with local and global deformations: deformable superquadrics. IEEE Transactions on Pattern Analysis and Machine Intelligence, 13(7):703-714, July 1991.

[60] B. Vemuri and A. Radisavljevic. Multiresolution stochastic hybrid shape models with fractal priors. ACM Transactions on graphics, 13(2):177-207, April 1994.

[61] B.C. Vemuri and A. Radisavljevic. From global to local, a continuum of shape models with fractal priors. Technical Report TR-92-036, Computer and Information Sciences Department - University of Florida, November 1992.

[62] Z. Zhang. Iterative point matching for registration of free-form curves and surfaces. International Journal of Computer Vision, 13(2):119-152, 1994. Also Research Report No.1658, INRIA SophiaAntipolis, 1992. 